\title{
L'INSEGNAMENTO DELLA COSTITUZIONE FEDERALE NEL CONTESTO DEL LICEO INTEGRATO DELL'ISTITUTO FEDERALE DI ISTRUZIONE, SCIENZA E TECNOLOGIA DI AMAPÁ (IFAP)
}

\section{ARTICOLO ORIGINALE}

COSTA, Pedro Henrique Maia1 ${ }^{1}$, COSTA, Susane de Almeida Aranha², FECURY, Amanda Alves ${ }^{3}$, DENDASCK, Carla Viana ${ }^{4}$, OLIVEIRA, Euzébio de ${ }^{5}$, DIAS, Claudio Alberto Gellis de Mattos ${ }^{6}$

COSTA, Pedro Henrique Maia. Et. L'insegnamento della Costituzione federale nel contesto del liceo integrato dell'Istituto federale di istruzione, scienza e tecnologia di Amapá (IFAP). Revista Científica Multidisciplinar Núcleo do Conhecimento. Anno 06, Ed. 04, Vol. 10, pp. 123-139. aprile 2021. ISSN: 2448-0959, Link di accesso: https://www.nucleodoconhecimento.com.br/formazioneit/integralizzato-dellistituto, DOI: 10.32749/nucleodoconhecimento.com.br/formazione-it/integralizzato-dellistituto

\section{RIEPILOGO}

La nostra Costituzione federale del 1988 ha inserito l'istruzione come diritto fondamentale nell'elenco dei diritti sociali. Uno degli scopi dell'istruzione è quello di garantire e preparare lo studente all'esercizio del cittadino. Le proposte evidenziate

\footnotetext{
${ }^{1}$ Ingegnere Civile, Professore e ricercatore presso l'Istituto di Educazione Di Base, Tecnica e Tecnologica di Amapá (IFAP), studente del Lato Sensu Graduate Program in Professional Education (IFAP).

${ }^{2}$ Avvocato, professore di lettere, post-laurea in metodologia dell'insegnamento della lingua inglese (UNITER) e diritto civile e commerciale (DAMASO).

${ }^{3}$ Biomedicale, Dottorato di Ricerca in Malattie Topiche, Professore e ricercatore del Corso di Medicina del Campus Macapá, Università Federale di Amapá (UNIFAP).

${ }^{4}$ Teologo, Dottore di Ricerca in Psicoanalisi, ricercatore presso il Centro di Ricerca e Studi Avanzati - CEPA.

${ }^{5}$ Biologo, Dottore di Ricerca in Malattie Topiche, Professore e ricercatore del Corso di Educazione Fisica, Università Federale di Pará (UFPA).

${ }^{6}$ Biologo, Dottore di Ricerca in Teoria e Comportamento, Professore e ricercatore del Corso di Laurea in Chimica dell'Istituto di Educazione Di Base, Tecnica e Tecnologica di Amapá (IFAP) e del Corso di Laurea in Formazione Professionale e Tecnologica (PROFEPT IFAP).
}

RC: 83562

Disponível em: https://www.nucleodoconhecimento.com.br/formazioneit/integralizzato-dellistituto 
non hanno lo scopo di formare una laurea in giurisprudenza, ma piuttosto un cittadino consapevole, che riconosce i suoi diritti e doveri fondamentali davanti alla società e allo Stato. L'obiettivo di questa ricerca è verificare la reale possibilità di introdurre lo studio della Costituzione Federale (CF) nell'IFAP, sulla base della conoscenza di questi studenti delle conoscenze di base sulla Costituzione federale. Questionari strutturati sono stati utilizzati con domande chiuse e aperte, di carattere dissertazione e altre di carattere oggettivo, applicate a gennaio 2018 ai partecipanti coinvolti, con l'aiuto di Google Forms. II liceo brasiliano, esaminato da un taglio puntuale del corso tecnico negli edifici nella forma integralizzata dell'IFAP, campus Macapá, non può rispettare le raccomandazioni del CF e della LDB in base al risultato del questionario applicato agli studenti. Questo insegnamento occupa un posto privilegiato nella formazione educativa brasiliana, tra le elementari e le superiori. Gli studenti dimostrano di essere entusiasti della possibilità di implementare scansioni di base nel corso tecnico negli edifici dell'IFAP, nel campus di Macapá, anche se facoltativamente.

Parole chiave: EPT, Legge, Istruzione, Costituzione, Scuola Superiore.

\section{INTRODUZIONE}

La nostra Costituzione federale del 1988 ha inserito l'istruzione come diritto fondamentale nell'elenco dei diritti sociali, consacrare costituzionalmente la sua importanza per la formazione e la vita nella società. L'educazione di una popolazione è una condizione indispensabile per lo sviluppo di uno Stato sia in ambito sociale, culturale, economico e scientifico (BRASIL, 2012).

La Costituzione della Repubblica Federativa del Brasile 1988 (CFRB/1988) prescrive, all'articolo 1, che: "la Repubblica Federativa del Brasile, formata dall'unione indissolubile di stati e comuni e dal Distretto Federale, costituisce uno Stato di Diritto Democratico e ha come fondamenta: II - cittadinanza;[...]" (BRASILE, 2012).

RC: 83562

Disponível em: https://www.nucleodoconhecimento.com.br/formazioneit/integralizzato-dellistituto 
Secondo ANTONELLO e NOREMBERG (2016), la cittadinanza è uno status in cui, se il suo obiettivo principale è quello di raggiungere la pienezza dell'esercizio dei diritti fondamentali inerenti all'individuo, uno status intrinsecamente legato al regime politico, poiché, sarà attraverso questo che il cittadino, attraverso i suoi rappresentanti, raggiungerà il suo diritto alle politiche pubbliche, cioè un'istruzione esemplare, salute, sicurezza, tra gli altri.

La legge sulle linee guida e le basi dell'istruzione brasiliana (LDB 9394/96) è la legislazione che regola il sistema educativo (pubblico o privato) del Brasile, dall'istruzione di base all'istruzione superiore. Uno degli scopi dell'Educazione, è quello di garantire e preparare lo studente all'esercizio del cittadino, l'idea di questo articolo è quella di discutere il modo migliore per raggiungere questo scopo, basando e giustificando tutte le posizioni assunte (BRASIL, 2016).

II 16 febbraio 2017, l'attuale presidente Michel Temer ha approvato la legge $\mathrm{n}$. 13.415 che riforma il liceo, ma questa riforma dipenderà direttamente dallo sviluppo della nuova Common National Curriculum Base (BNCC), che dovrebbe essere approvata entro la fine dell'anno 2018. il nuovo liceo è stato modificato attraverso misure provvisorie (MP) rigorosamente seguendo i requisiti previsti dalla Costituzione federale, ma alcuni esperti considerano questa forma di azione una battuta d'arresto sociale, poiché i cambiamenti avrebbero dovuto essere apertamente discussi con la società e non attuati tramite MP (BRASIL, 2017).

La legge sulle linee guida e le basi dell'istruzione brasiliana (LDB 9394/96), negli articoli 2 e 22, stabilisce che l'istruzione mira al pieno sviluppo dello studente e alla sua preparazione all'esercizio della cittadinanza, garantita attraverso l'istruzione di base. Ma per raggiungere questo obiettivo, è essenziale democratizzare la conoscenza giuridica all'interno della società (BRASIL, 2016).

Nella ricerca di questo successo, attualmente, la legge è stata osservata con altri occhi, non più con una visione positivista e ristretta, ma ora come una conoscenza necessaria e indispensabile per la vita dell'individuo. Tuttavia, è palpabile l'ignoranza

RC: 83562

Disponível em: https://www.nucleodoconhecimento.com.br/formazioneit/integralizzato-dellistituto 
che una parte significativa del popolo brasiliano presenta in relazione alle basi della Costituzione federale, questa percezione è stata confermata da una ricerca datasenate realizzata esclusivamente per un'edizione speciale nel 2013 (SASSE, 2013)

Secondo MORAES (2013), "ci sono numerose ragioni per includere le materie giuridiche nel liceo. Ma la cosa fondamentale è formare un cittadino, individui che formano opinioni, ben informata dei loro diritti e doveri.Tuttavia, se l'intenzione è la corretta formazione dei cittadini attraverso la conoscenza giuridica, è prudente dimostrare che, in un certo senso, questa idea viene già rispettata in Brasile, anche in modo incipiente e puntuale.

Lo Statuto del Bambino e dell'Adolescente (ECA) è stato istituito con la legge $n$. 8.069 il 13 luglio 1990. Disciplina i diritti e prevede la piena protezione dei bambini e degli adolescenti ispirati ai principi costituzionali. Sebbene la Corte dei conti europea sia una legge in vigore dal 1990, vi sono state una serie di difficoltà e sfide per la sua attuazione nella società brasiliana. I principali responsabili della tutela e dell'applicazione dei diritti dei bambini e degli adolescenti sono, oltre alla società in generale, la famiglia e le autorità pubbliche (SARAIVA, 2017).

L'Ordine degli avvocati brasiliano (OAB) ha creato un progetto di insegnamento per le scuole superiori in Brasile, chiamato OAB goes to school che è iniziato nel 1993 su iniziativa del consigliere di OAB SP e presidente della Commissione di cittadinanza e azione sociale, all'epoca, Nelson Alexandre da Silva Filho, nella città di Osasco, e ampliato in tutto il Brasile , che copre circa 20 milioni di alunni delle scuole pubbliche. Diverse scuole pubbliche in Brasile sono state contemplate dal progetto, con I'obiettivo di chiarire il ruolo dello stesso OAB nello Stato e il suo funzionamento, oltre a contribuire alla cittadinanza degli studenti attraverso lezioni frontali, opuscoli, film educativi e, naturalmente, con dibattiti che stimolano il loro interesse per le conoscenze giuridiche. (SILVA FILHO, 2004).

RC: 83562

Disponível em: https://www.nucleodoconhecimento.com.br/formazione- 
DIAS e DE OLIVEIRA (2015) presentano alcuni suggerimenti riguardanti i contenuti di base del diritto che possono essere integrati nell'educazione regolare, vale a dire i concetti di Diritti Umani, Lo Statuto dei bambini e degli adolescenti, i diritti dei consumatori, anche con particolare attenzione al mercato elettronico, all'educazione fiscale e anche ai percorsi di giustizia.

MORAES (2013) ritiene che sarebbe di grande valore sociale includere nelle scuole superiori le seguenti discipline: Introduzione al diritto, diritti costituzionali e garanzie, diritto penale, diritto civile e leggi sparse, come lo studio di alcune leggi come lo Statuto dei bambini e degli adolescenti. Lo studente avrebbe anche bisogno di avere conoscenze di base sulla Pubblica Amministrazione, sulla gerarchia dei poteri, sulla creazione e promulgazione delle leggi.

Le proposte evidenziate non hanno lo scopo di formare una laurea in giurisprudenza, ma piuttosto un cittadino consapevole, che riconosce i suoi diritti e doveri fondamentali davanti alla società e allo Stato.

\section{OBIETTIVO}

Verificare la reale possibilità di introdurre lo studio della Costituzione Federale (CF) nell'IFAP, sulla base della conoscenza di questi studenti delle conoscenze di base sulla Costituzione Federale.

\section{METODO}

Questionari strutturati sono stati utilizzati con domande chiuse e aperte, di carattere dissertazione e altre di carattere oggettivo, applicate a gennaio 2018 ai partecipanti coinvolti, con l'aiuto di Google Forms.

Il pubblico di riferimento era composto da 20 membri della facoltà del corso tecnico collegiale in costruzione nella forma integralizzata, pedagoghi e coordinatori di corsi tecnici in forma integralizzata, il direttore dell'insegnamento e il direttore generale 
dell'IFAP, campus Macapá, oltre ad alcuni avvocati appartenenti all'OAB nello stato di Amapá, dipendenti dell'IFAP stesso. Un altro questionario con domande chiuse, di carattere oggettivo, è stato applicato a 106 studenti dei corsi tecnici degli edifici Ifap nel campus di Macapá senza preavviso, suddivisi in cinque sezioni per verificare il grado di conoscenza che hanno presentato in relazione alla visibilità sociale dei tre poteri in ambito comunale, al figlio fondamentale della Costituzione federale e in relazione ai principi di giustizia, derivanti dai costumi e/o dal buon senso di una società.

\section{RISULTATIE DISCUSSIONE}

Sia l'analisi quantitativa che qualitativa hanno cercato di raggruppare le domande per sezioni specifiche di punti da esaminare per facilitare l'interpretazione dei dati e la discussione dei risultati.

\section{QUESTIONARIO APPLICATO AL PERSONALE TECNICO IFAP}

Analizzando la figura 01, possiamo osservare che la maggior parte dei partecipanti a questo questionario ha trent'anni, la maggior parte dei quali ha 32 anni. Questo risultato è dovuto al fatto che ifap è un istituto recente, dove c'è il costante ingresso di professionisti per soddisfare la crescente domanda del pubblico di destinazione (IFAP, 2021). 
Figura 01 Numero intervistato in base all'età.

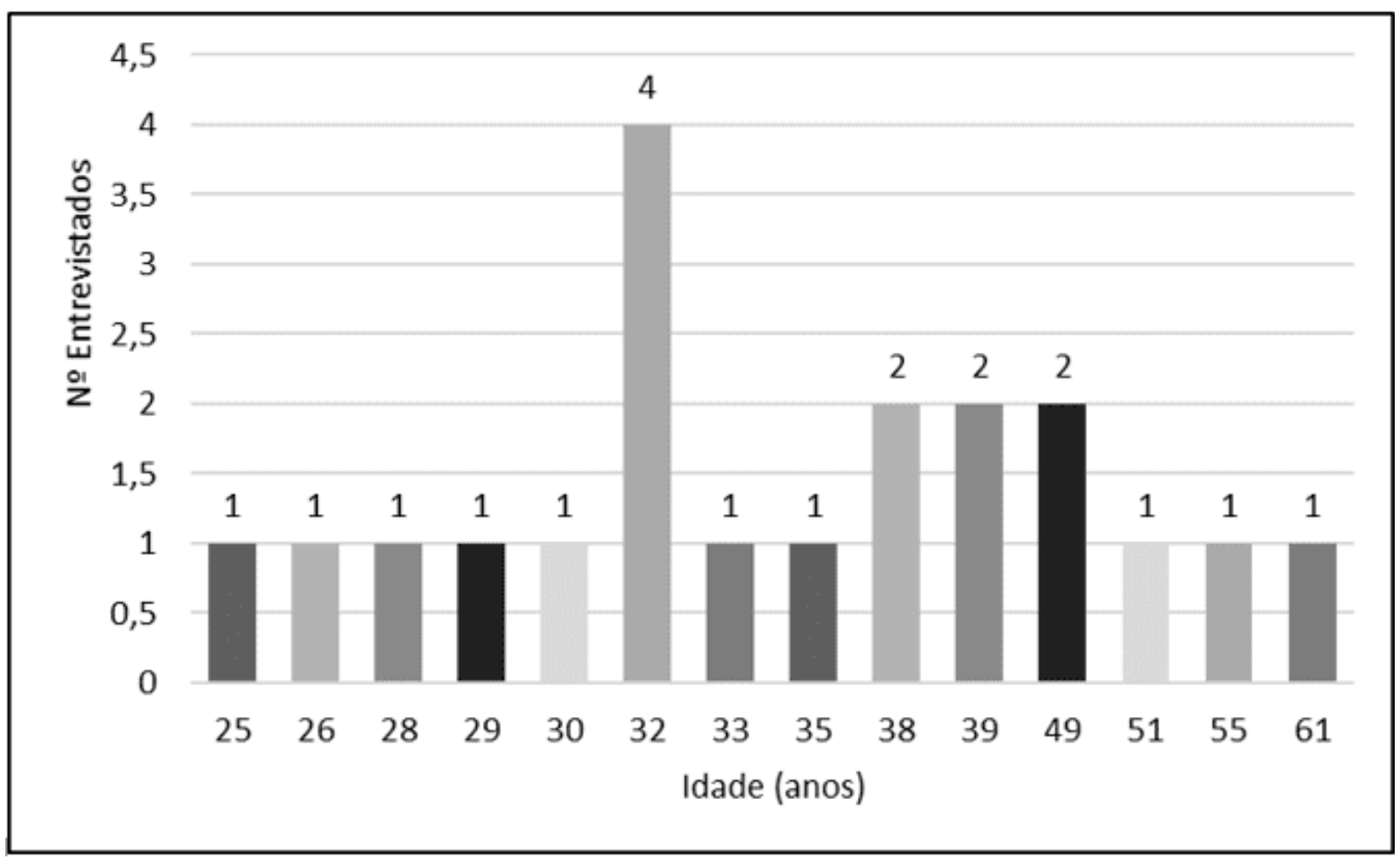

RC: 83562

Disponível em: https://www.nucleodoconhecimento.com.br/formazioneit/integralizzato-dellistituto 
Figura 02: Percentuale di intervistati in base al sesso.

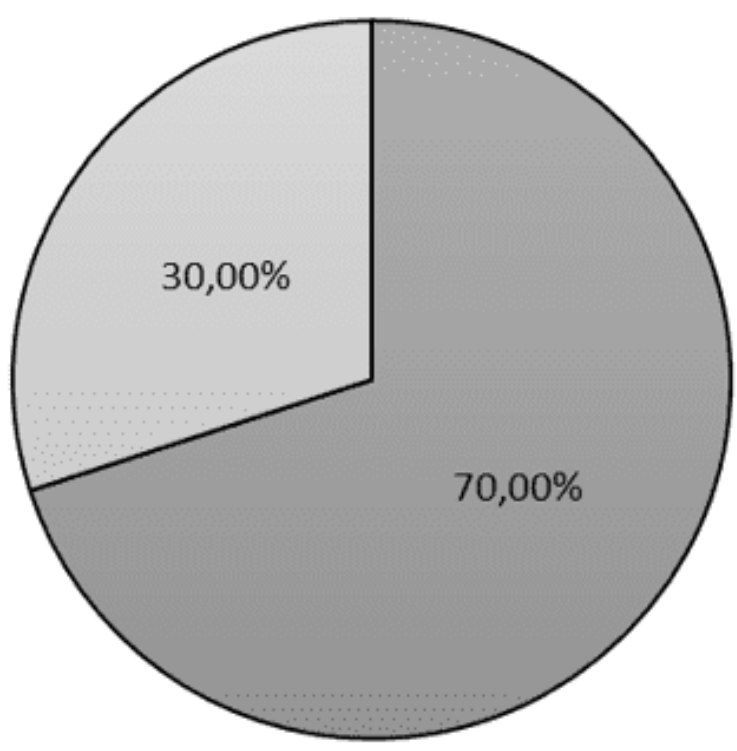

$\square$ Masculino $\square$ Feminino

La figura 02 mostra un numero più elevato di intervistati maschi, più del doppio delle donne, un fatto che non è stato evidenziato nei risultati del questionario applicato agli studenti, che ha dimostrato una certa omogeneità in relazione al genere. Questo è un riflesso del maggior numero di uomini nel mercato del lavoro brasiliano (NÓBREGA et al., 2019).

Per quanto riguarda la professione/posizione, la Figura 03 presenta un risultato atteso, in cui il maggior numero di intervistati sono stati docenti della base didattica nazionale comune del corso tecnico negli edifici, poiché le discipline del BNCC accumulano circa il 69\% del carico di lavoro totale del corso (IFAP, 2019). 
Figura 03 Numero intervistato in base alla professione/posizione.

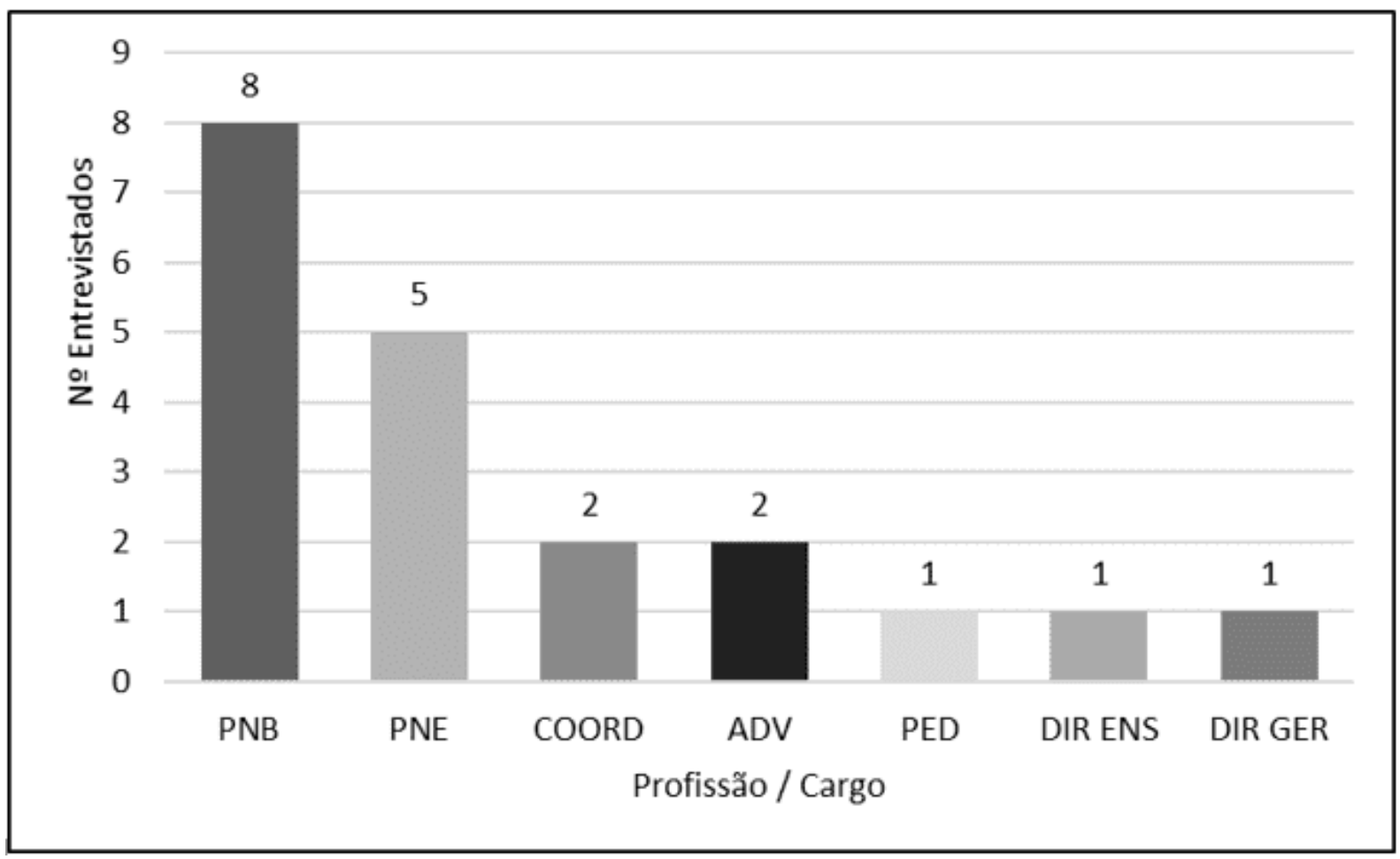

RC: 83562

Disponível em: https://www.nucleodoconhecimento.com.br/formazioneit/integralizzato-dellistituto 
Figura 04 Numero intervistato in base al tempo dell'esperienza professionale.

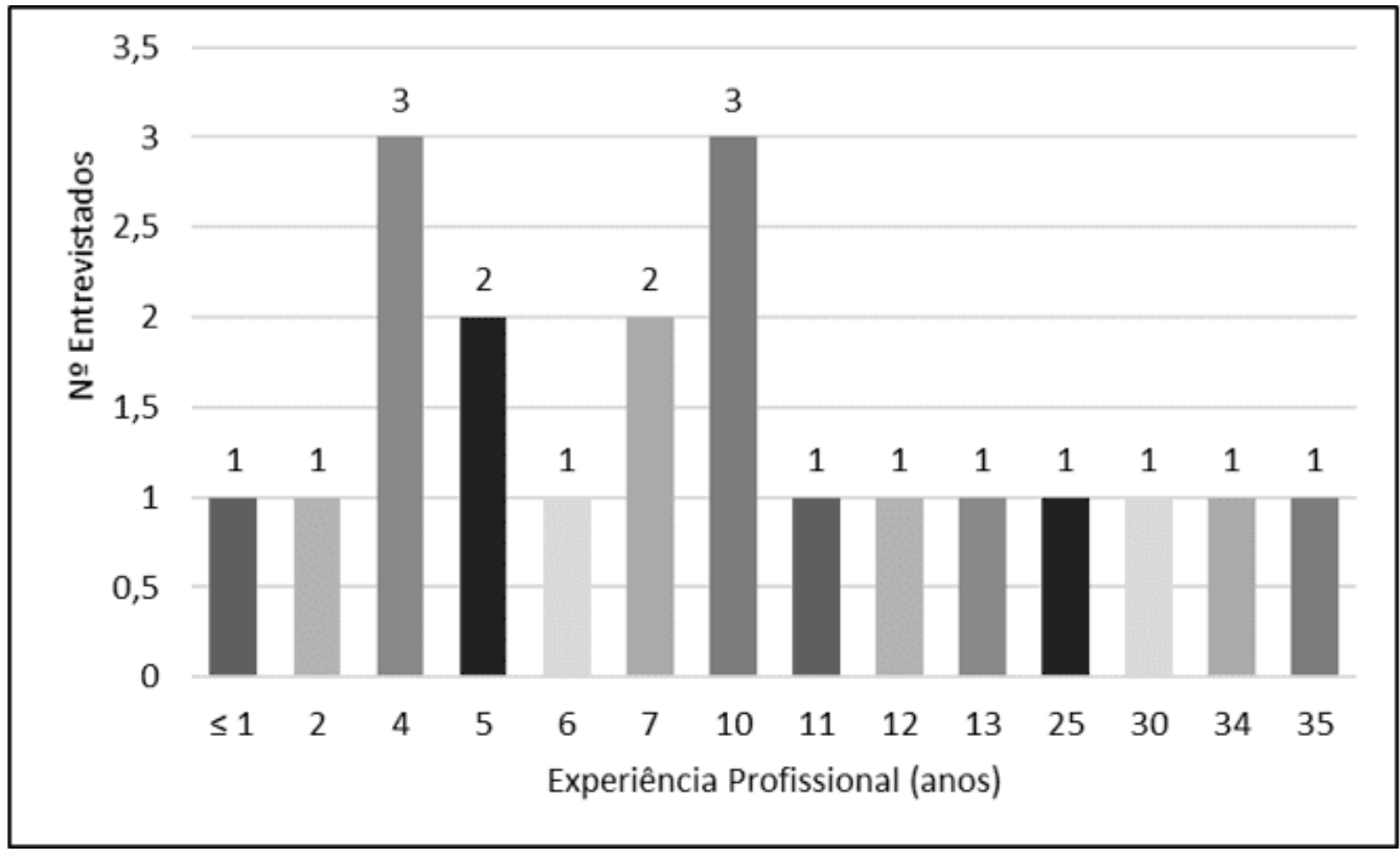

Nella figura 04 , notiamo che la maggior parte degli intervistati non ha una vasta esperienza professionale, anche se i risultati sono considerati molto rilevanti, perché tutti gli intervistati sono dipendenti pubblici che non hanno bisogno di omettere 0 distorcere le risposte per motivi di altri (BRASIL, 1990).

Gli intervistati, alla domanda se avessero già letto o maneggiato (impiallacciato) il $\mathrm{CF}$, il $90 \%$ ha risposto positivamente, come evidenziato nella figura 05 e la maggioranza ha riferito che la parte che ha attirato la loro attenzione erano i "Diritti e garanzie fondamentali", in particolare l'articolo 5 e il diritto sociale allistruzione, elencati nell'articolo 6 , dimostrando che la maggior parte dei professionisti accede alla Carta Magna per conoscere i loro diritti e garanzie e , logicamente, sul settore dell'istruzione, poiché la maggior parte degli intervistati sono insegnanti e lavorano nell'area didattica (BRASIL, 1990). 
Figura 05 Percentuale di intervistati che hanno letto o gestito (sfogliato) il CF.

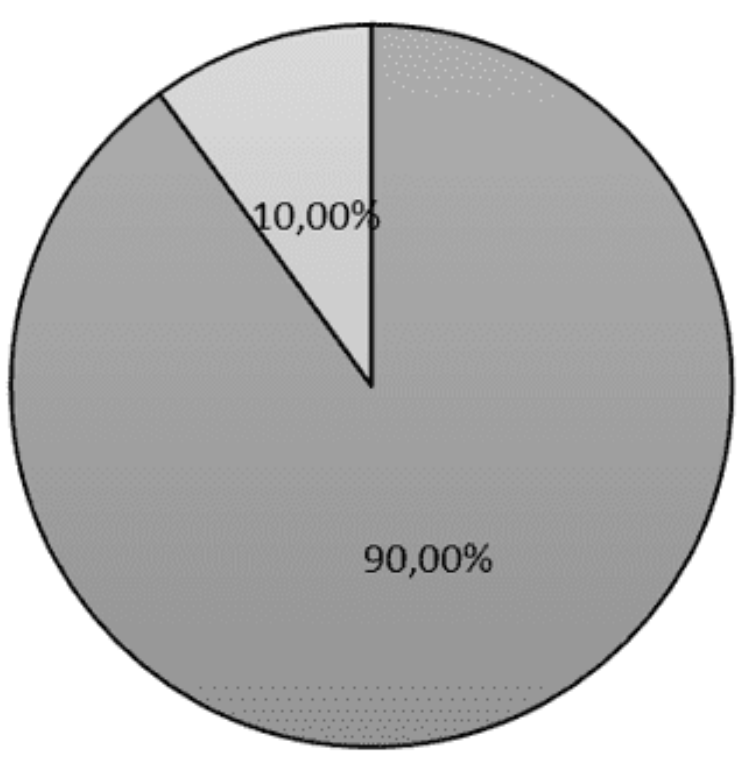

$\square \operatorname{Sim} \square$ Não

Per quanto riguarda il grado di conoscenza che i partecipanti all'intervista ritengono di avere in relazione al CF del 1988, il 55\% di loro ritiene di non avere conoscenze sufficienti per il pieno esercizio della cittadinanza, il 40\% considera di avere conoscenze sufficienti e un solo avvocato ha ritenuto di avere un'ottima conoscenza della nostra Costituzione federale, cosa che era da aspettarsi, perché agisce direttamente con l'argomento richiesto, questo risultato può essere evidenziato nelle figure 06, il che dimostra un equilibrio sul grado di conoscenza dei partecipanti in relazione a CF (FONSECA e FERNANDES, 2020). 
Figura 06 Grado di conoscenza in relazione al CF 1988 - A) numero di intervistati; B) percentuale di intervistati.

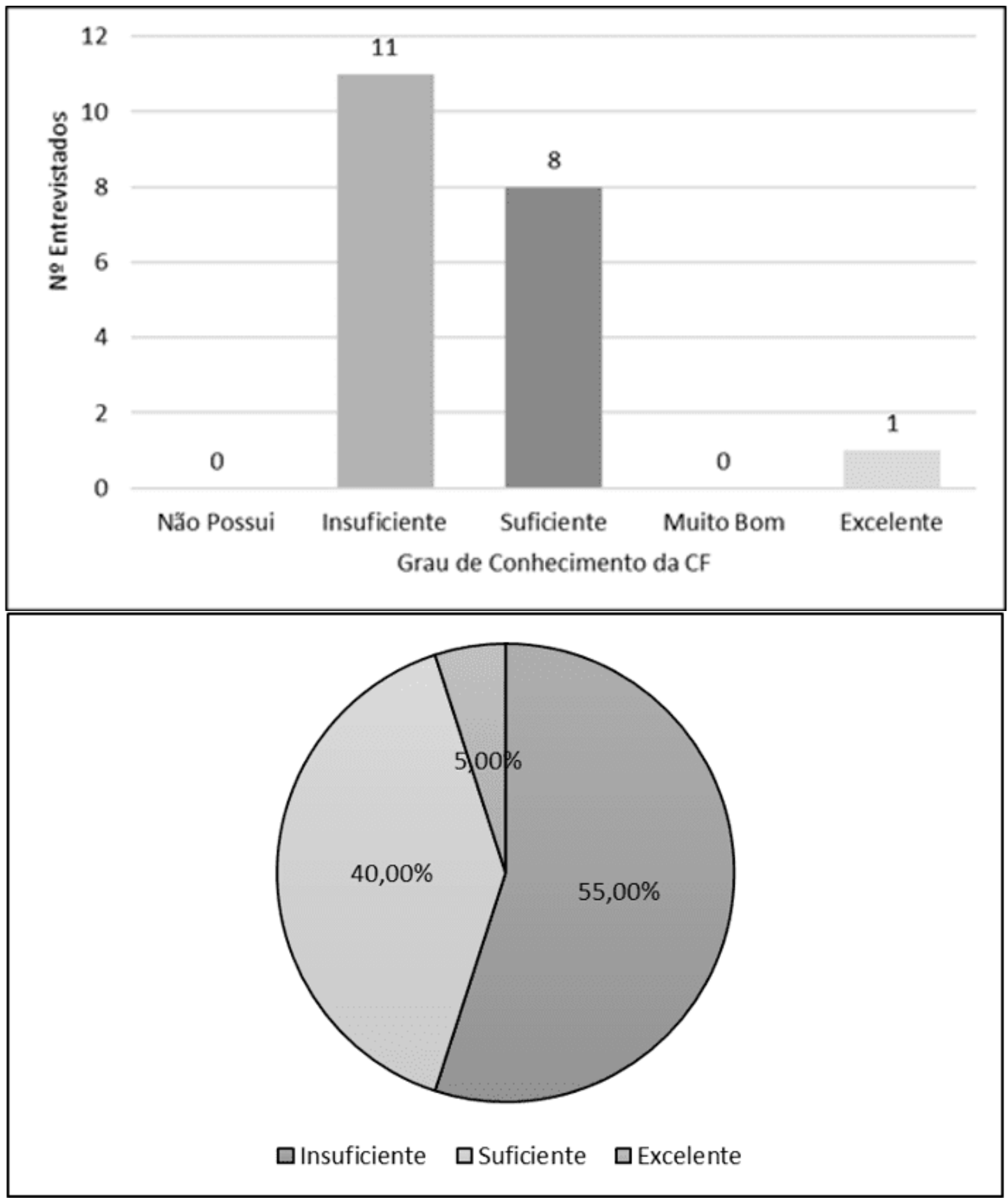

RC: 83562

Disponível em: https://www.nucleodoconhecimento.com.br/formazioneit/integralizzato-dellistituto 
Interrogata sulla pertinenza dell'insegnamento delle basi della Costituzione federale nell'attuale istruzione di base, la stragrande maggioranza ha ritenuto questo insegnamento estremamente importante per gli studenti anche se nella sua forma di base, contribuendo alla crescita intellettuale e umanistica degli studenti (LORENSET et al., 2021).

Per quanto riguarda la conoscenza di alcune proposte di legge nel tentativo di formalizzare l'insegnamento del diritto nelle scuole, solo il $20 \%$ ha risposto per avere conoscenza (figura 07) e ha citato principalmente il disegno di legge del senatore Romário, anche se superficialmente ha scoperto che questo disegno di legge è il più noto con la comunità accademica (ROMARIO, 2021).

Figura 07 Percentuale di intervistati che conoscono un disegno di legge nel tentativo di formalizzare l'insegnamento del diritto nelle scuole.

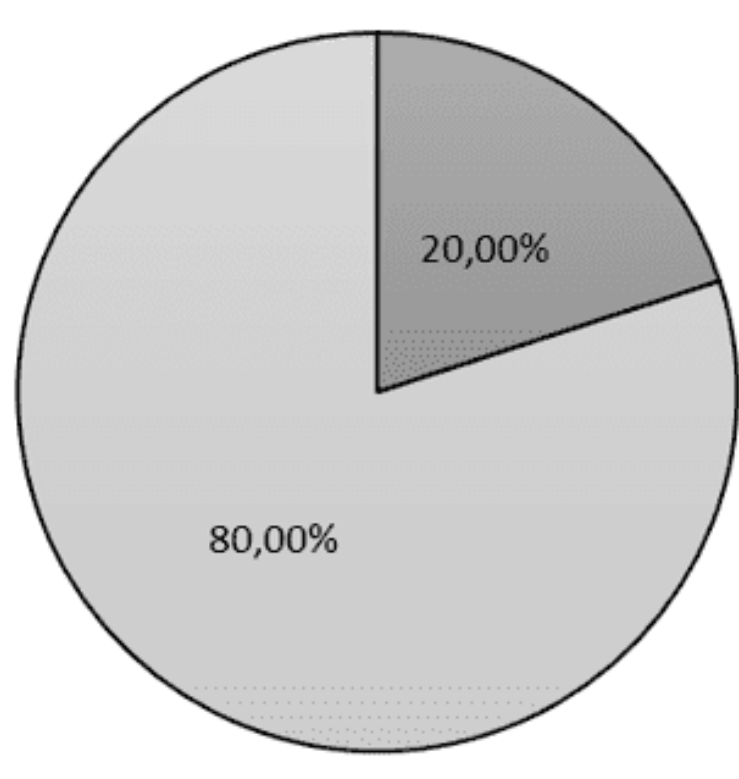

口Sim ロNão

Quando sono state affrontate le somme fondamentali della Costituzione federale che dovrebbero essere insegnate agli studenti dell'istruzione di base, le materie principali 
menzionate sono state: diritti e garanzie fondamentali, cittadinanza, principi fondamentali, statuto dei bambini e degli adolescenti e diritti umani. Dal punto di vista del $40 \%$ degli intervistati, questi contenuti dovrebbero essere studiati sia alle scuole elementari che alle superiori e, come mostrato nella figura 08 , nessun intervistato ha scelto l'alternativa di studiare questi contenuti solo nell'istruzione superiore, il che dimostra già una tendenza favorevole ad attuare questo insegnamento nell'istruzione di base (HOHENDORFF et al., 2020).

Figura 08 Parere sul palco secondo cui l'insegnamento delle basi della CF dovrebbe essere studiato - a) numero di intervistati; b) percentuale di intervistati.

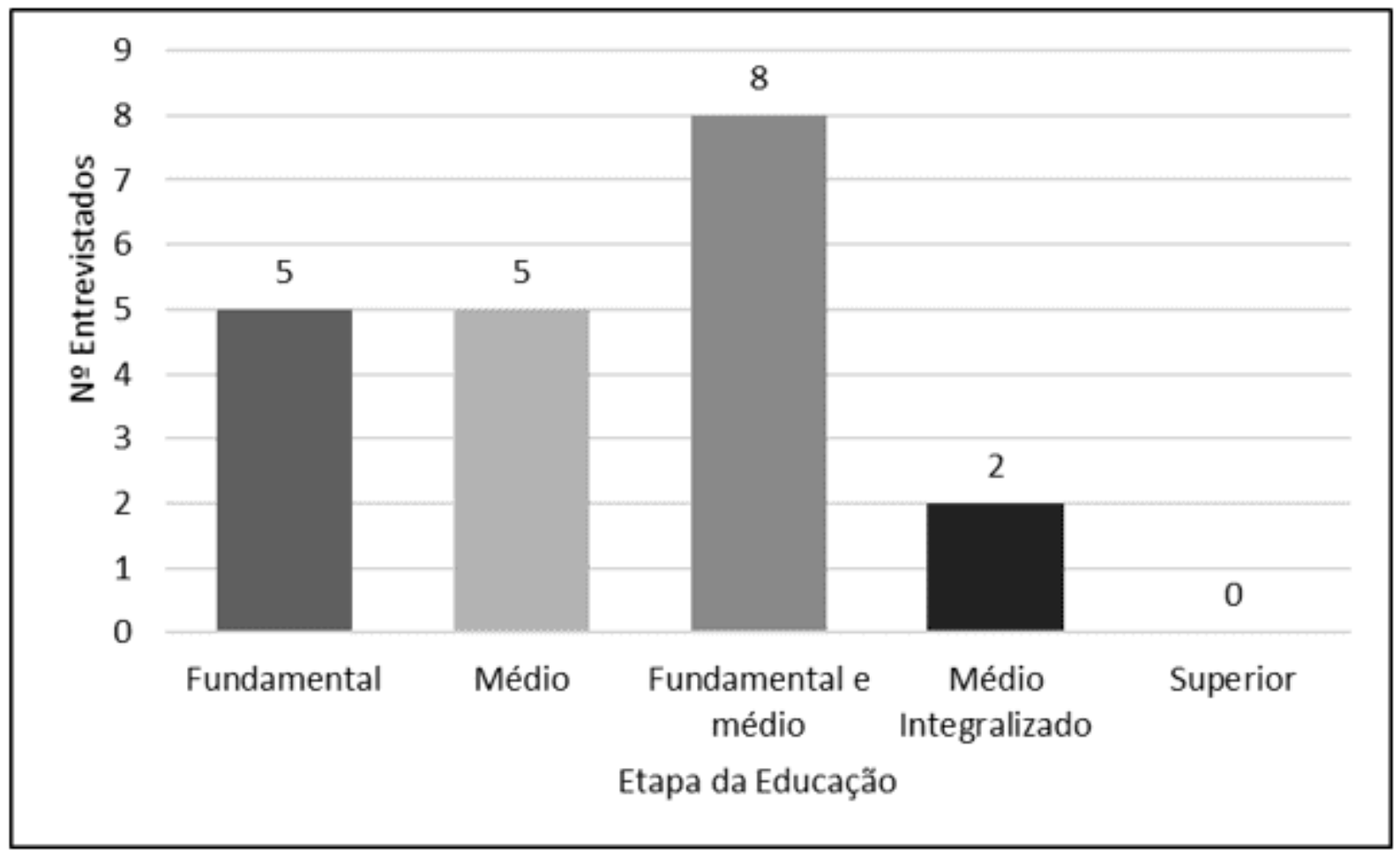

RC: 83562

Disponível em: https://www.nucleodoconhecimento.com.br/formazioneit/integralizzato-dellistituto 


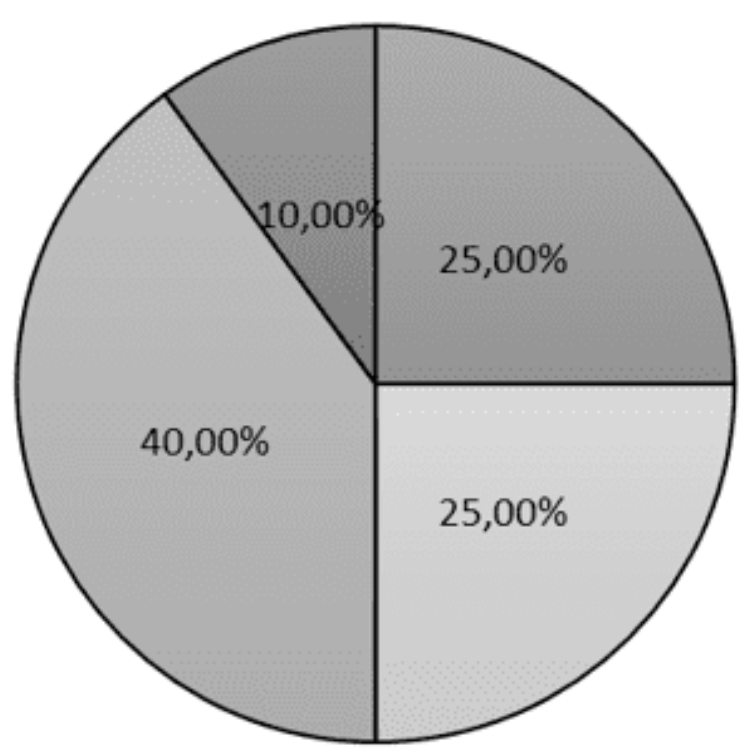

\section{$\square$ Fundamental $\square$ Médio $\square$ Fundamental e médio $\square$ Médio Integralizado}

Agli intervistati è stato chiesto se hanno avuto l'opportunità per tutta la vita accademica di studiare materie come l'Organizzazione sociale e politica del Brasile (OSPB) e l'educazione morale e civica (EMC), il 55\% degli intervistati non ha mai studiato tali materie e il $45 \%$ ha avuto l'opportunità di studiarle per tutta la vita accademica. Ciò riflette l'età degli intervistati, perché coloro che hanno più di 35 anni probabilmente hanno completato l'istruzione di base al momento del regime militare, un periodo in cui queste materie sono state ampiamente diffuse nell'istruzione di base nelle scuole pubbliche (AMARAL e CASTRO, 2020). La maggior parte degli intervistati che hanno risposto positivamente alla domanda ha sostenuto che queste discipline hanno contribuito alla formazione di un pensiero più critico, individui più consapevoli dei loro diritti e doveri e più preparati all'esercizio della cittadinanza (TAVARES et al., 2020).

Secondo la possibilità di attuare l'insegnamento delle somme di base della Costituzione federale nell'attuale scenario dell'istruzione brasiliana, solo due intervistati, un insegnante del nucleo comune e uno del nucleo specifico, si sono opposti a questa proposta, rivendicando un elevato carico di lavoro nell'istruzione di 
base e il fatto che questa proposta doveva partire dal Ministero dell'Istruzione (MEC) per essere attuata, altrimenti sarebbe irrealizzabile. D'altra parte, la maggior parte degli intervistati era favorevole alla proposta, sostenendo che i progressi tecnologici avrebbero facilitato questo insegnamento, oltre a promuovere un graduale cambiamento nell'attuale scenario politico brasiliano e un aumento della consapevolezza dei cittadini sui loro diritti e doveri (TAVARES et al., 2020).

\section{QUESTIONARIO APPLICATO AGLI STUDENTI DEL CORSO TECNICO IFAP}

La figura 9 mostra che la maggior parte degli studenti che hanno partecipato alla ricerca ha tra i 15 e i 18 anni, ciò è dovuto al fatto che gli intervistati sono studenti delle scuole superiori nella forma integralizzata che si adatta a questa fascia di età. Abbiamo anche rilevato che la maggior parte dei partecipanti aveva 15 anni, questo risultato è confermato dal grafico della Figura 10, che spiega che la maggior parte degli studenti che hanno risposto al questionario sono iscritti al $1^{\circ}$ anno, cioè stanno iniziando studenti delle scuole superiori con un'età media di 15 anni (IFAP, 2019). 
Figura 09 Distribuzione degli studenti partecipanti in base all'età.

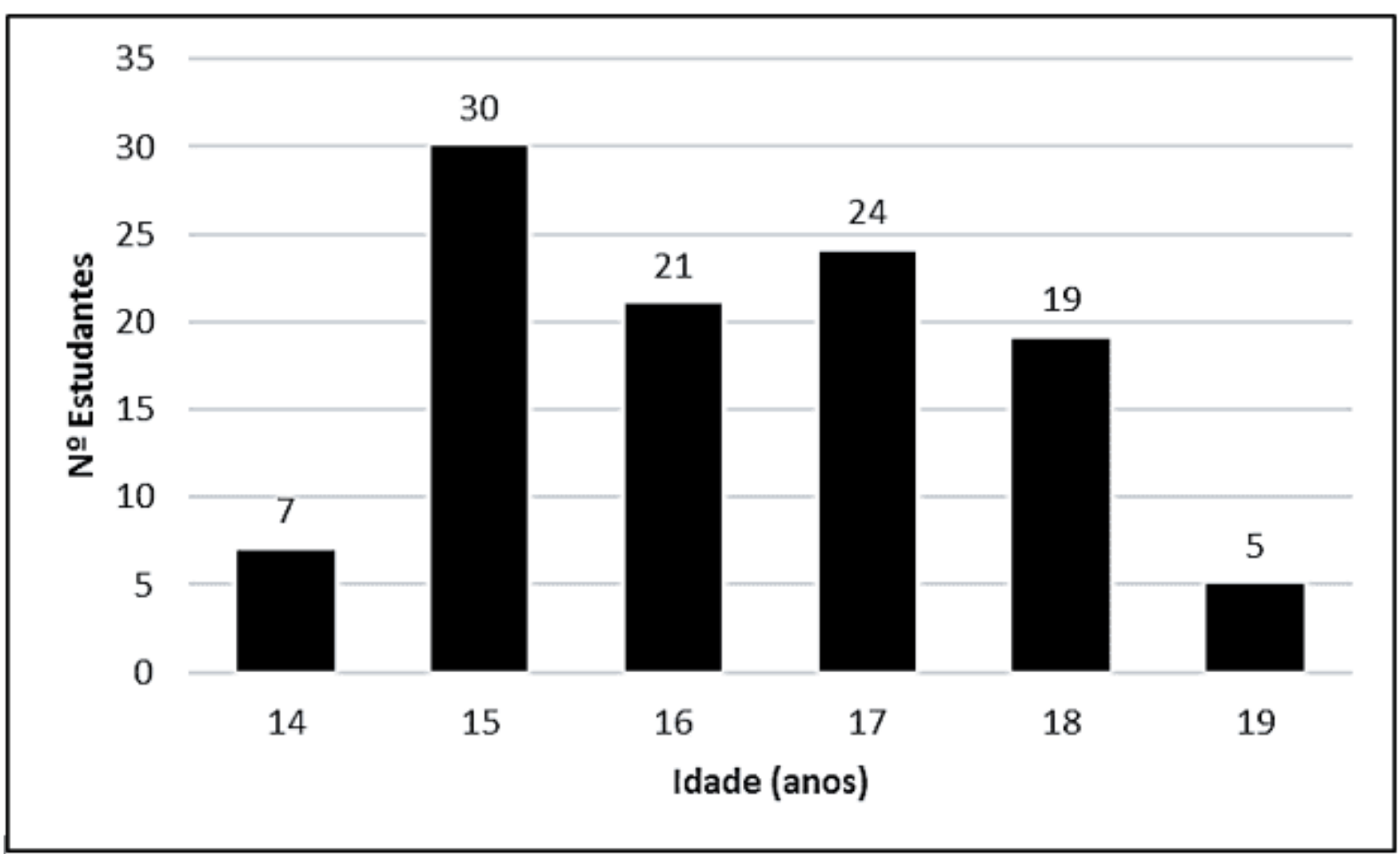

RC: 83562

Disponível em: https://www.nucleodoconhecimento.com.br/formazioneit/integralizzato-dellistituto 
Figura 10 Distribuzione degli studenti partecipanti in base al voto/anno.

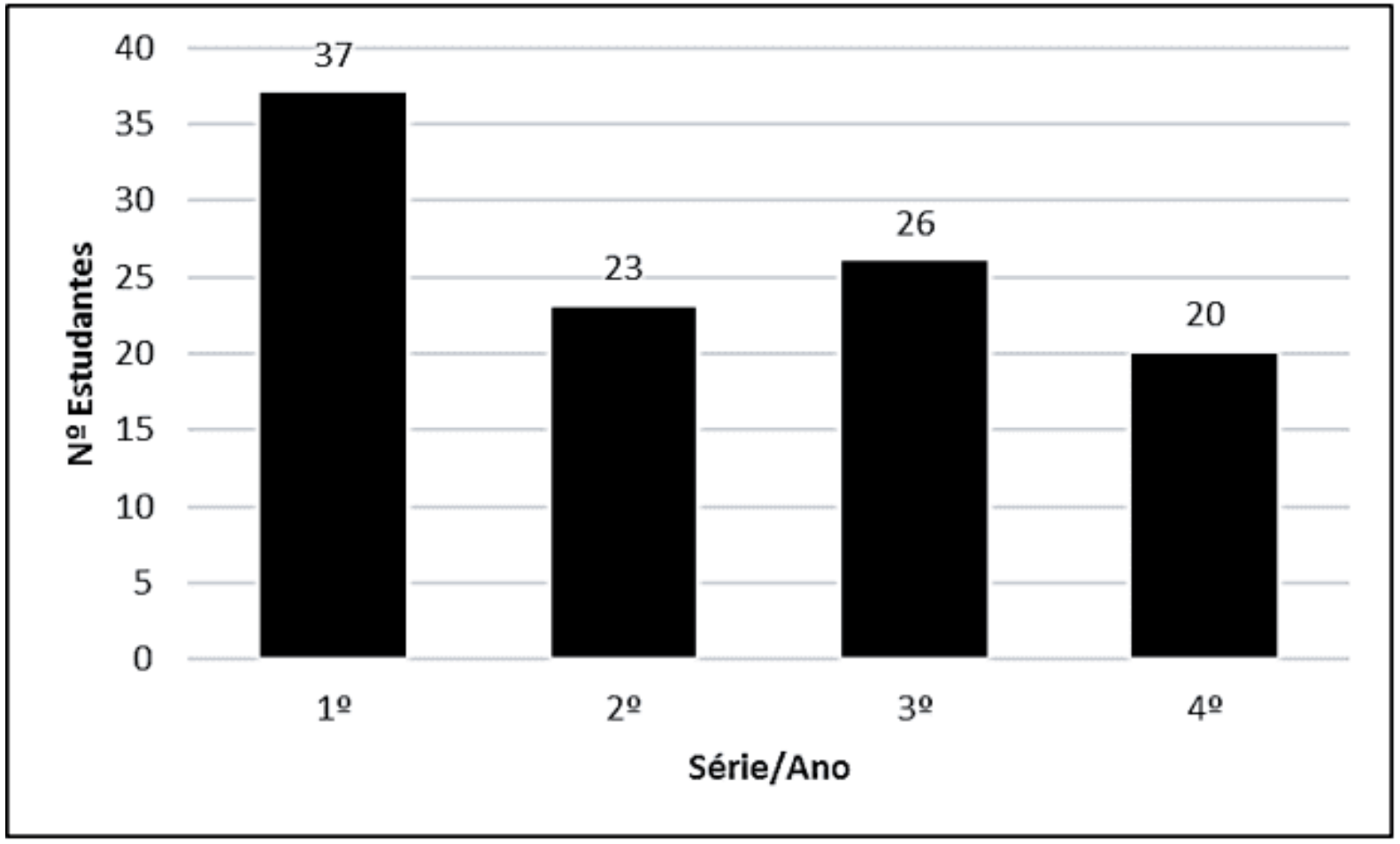

Dei 106 studenti che hanno partecipato alla ricerca, 56 sono maschi e 50 femmine, il che dimostra un equilibrio in relazione al sesso degli intervistati, questo risultato può essere evidenziato nella figura 11 , dove queste cifre mostrano che il $52,83 \%$ degli intervistati sono maschi e il 47,17\% sono femmine (IBGE, 2010).

Per quanto riguarda il reddito familiare, la figura 12 mostra che la maggior parte del pubblico intervistato appartiene alla classe povera. Questo è il pubblico del liceo nella forma integralizzata di ifap, indipendentemente dal campus o dal corso, riflettendo in modo simile la piramide dei redditi del Brasile (IBGE, 2021). 
Figura 11 Distribuzione degli studenti partecipanti in base al sesso.

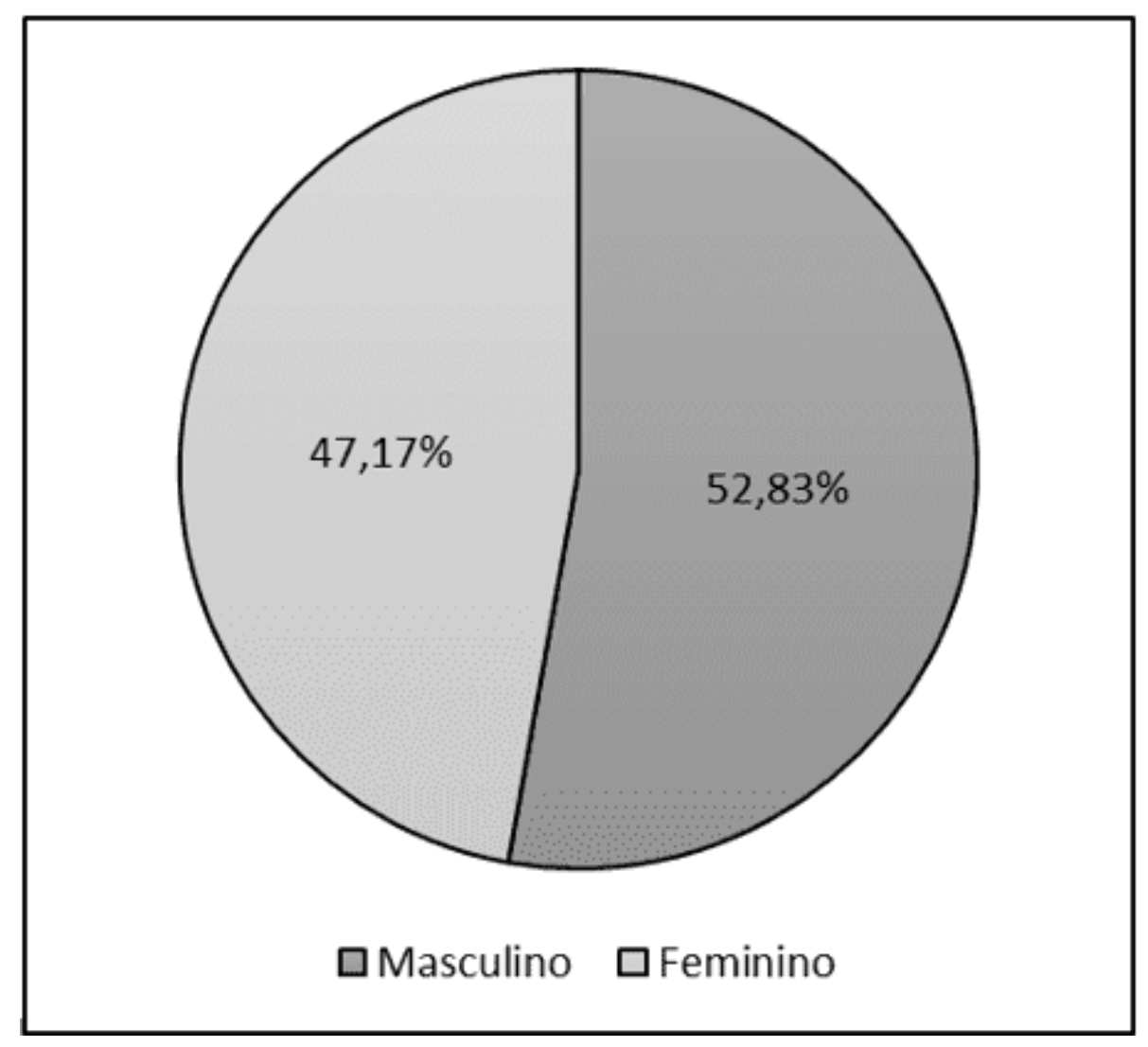

RC: 83562

Disponível em: https://www.nucleodoconhecimento.com.br/formazioneit/integralizzato-dellistituto 
Figura 12 Distribuzione degli studenti partecipanti in base al reddito familiare.

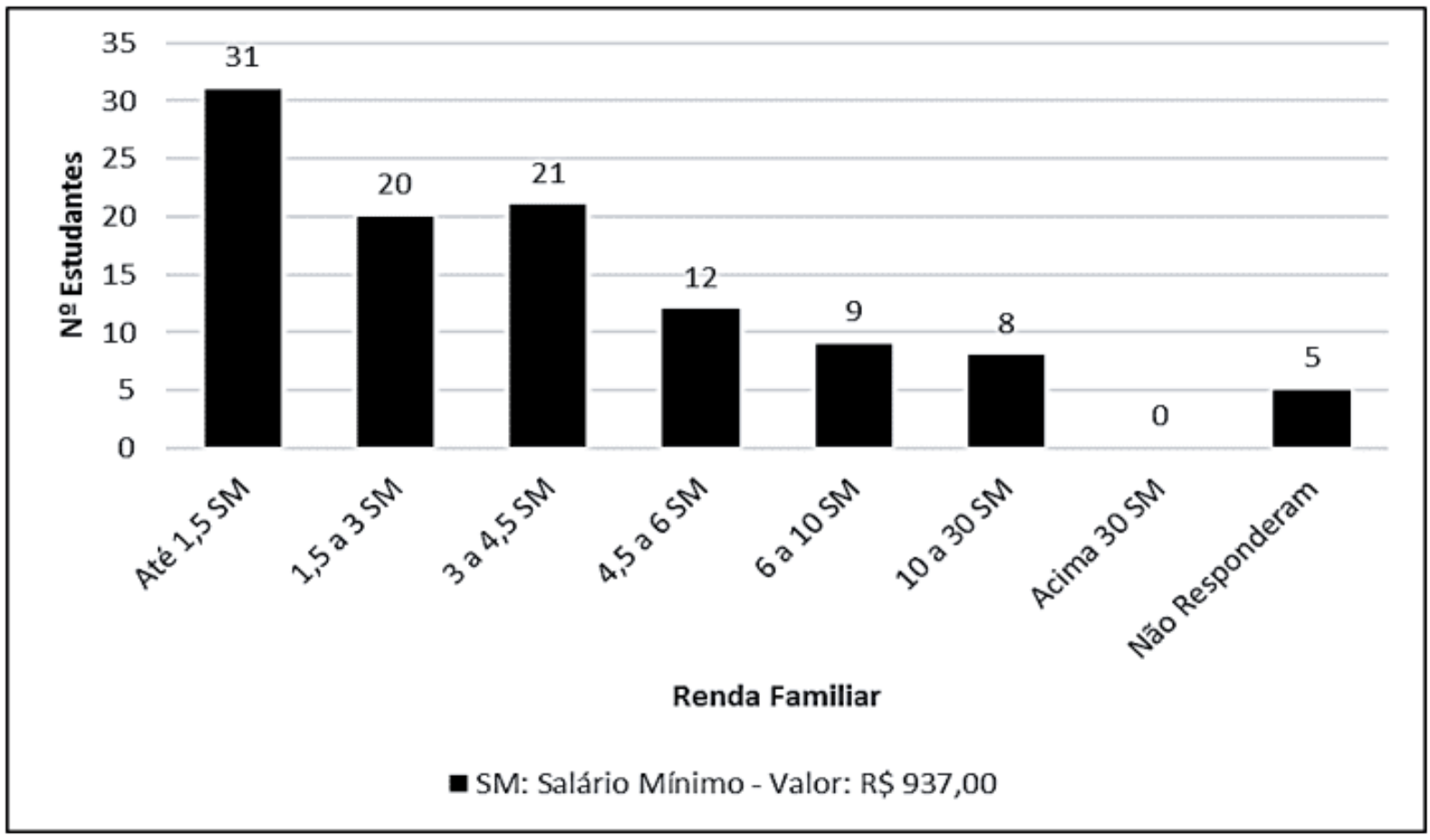

Si chiese della posizione spaziale delle tre potenze a Macapá. La conoscenza dell'ubicazione delle tre potenze nello spazio urbano è la prova della loro importanza sociale. II risultato ha dimostrato che l'esecutivo è il più visibile, seguito dal legislatore e infine dalla magistratura, come mostrato nella figura 13 , dove il $44,65 \%$ degli intervistati ha risposto correttamente alle domande sulla notorietà del ramo esecutivo. Questo riflesso della visibilità sociale dei tre poteri è dovuto a un maggiore investimento dell'esecutivo e del legislatore nei media rispetto alla magistratura, soprattutto perché i suoi occupanti sono eletti dal popolo, il che rende questa notorietà sempre più necessaria per la loro promozione personale, mentre gli occupanti della magistratura, per lo più , si insediano tramite gara d'appalto pubblica, cioè si basano sul principio della meritocrazia per detenere l'incarico e non richiedono il voto popolare (FIGUEIREDO, 2019). 
Figura 13 Visibilità sociale delle tre potenze costituenti a Macapá.

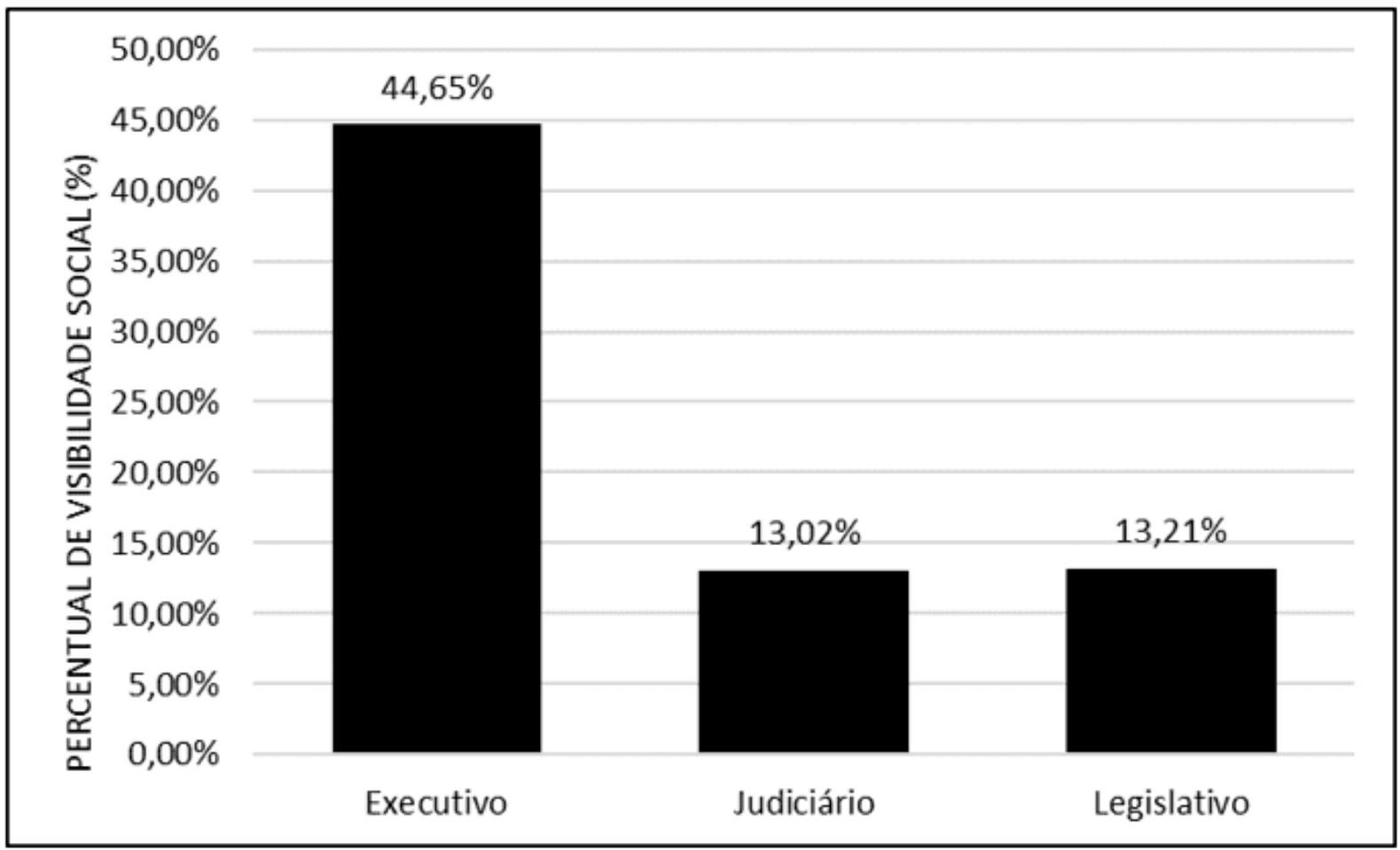

Le quattro figure seguenti (da 14 a 17) fanno una correlazione tra la visibilità sociale delle 3 potenze in Macapá e i fattori che potrebbero influenzare questo indice di notorietà. Tra i 4 fattori intervistati, quello che ha presentato una linea di tendenza più evidente è stato quello che ha correlato la visibilità sociale e la serie/anno dei partecipanti, mostrato nella figura 15 , dove è stato rilevato che la visibilità sociale delle 3 potenze aumenta man mano che gli studenti passavano al voto successivo. 
Figura 14 Visibilità sociale dei tre poteri costituenti a Macapá in base all'età.

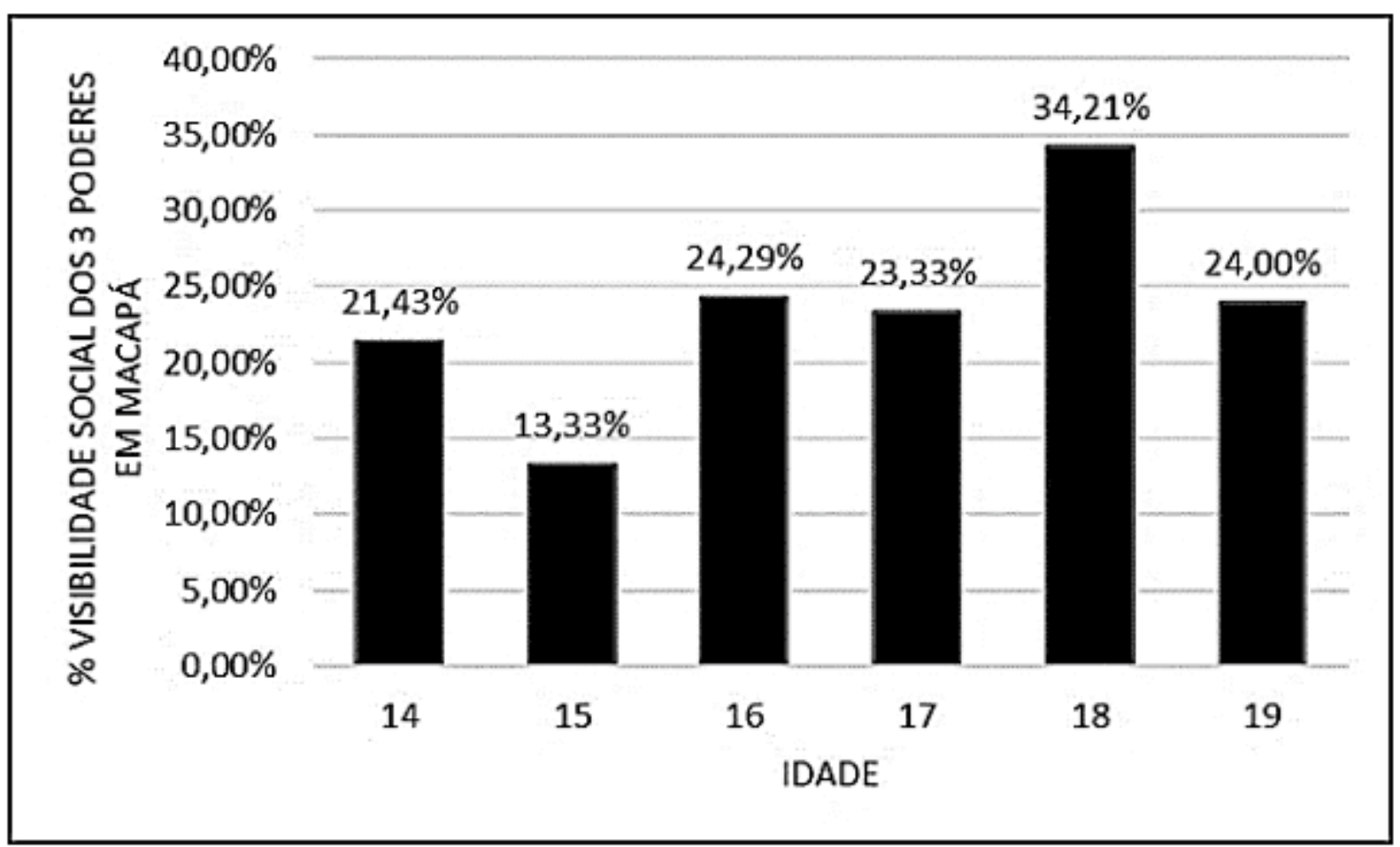

RC: 83562

Disponível em: https://www.nucleodoconhecimento.com.br/formazioneit/integralizzato-dellistituto 
Figura 15 Visibilità sociale delle tre potenze costituenti a Macapá a seconda della serie/anno.

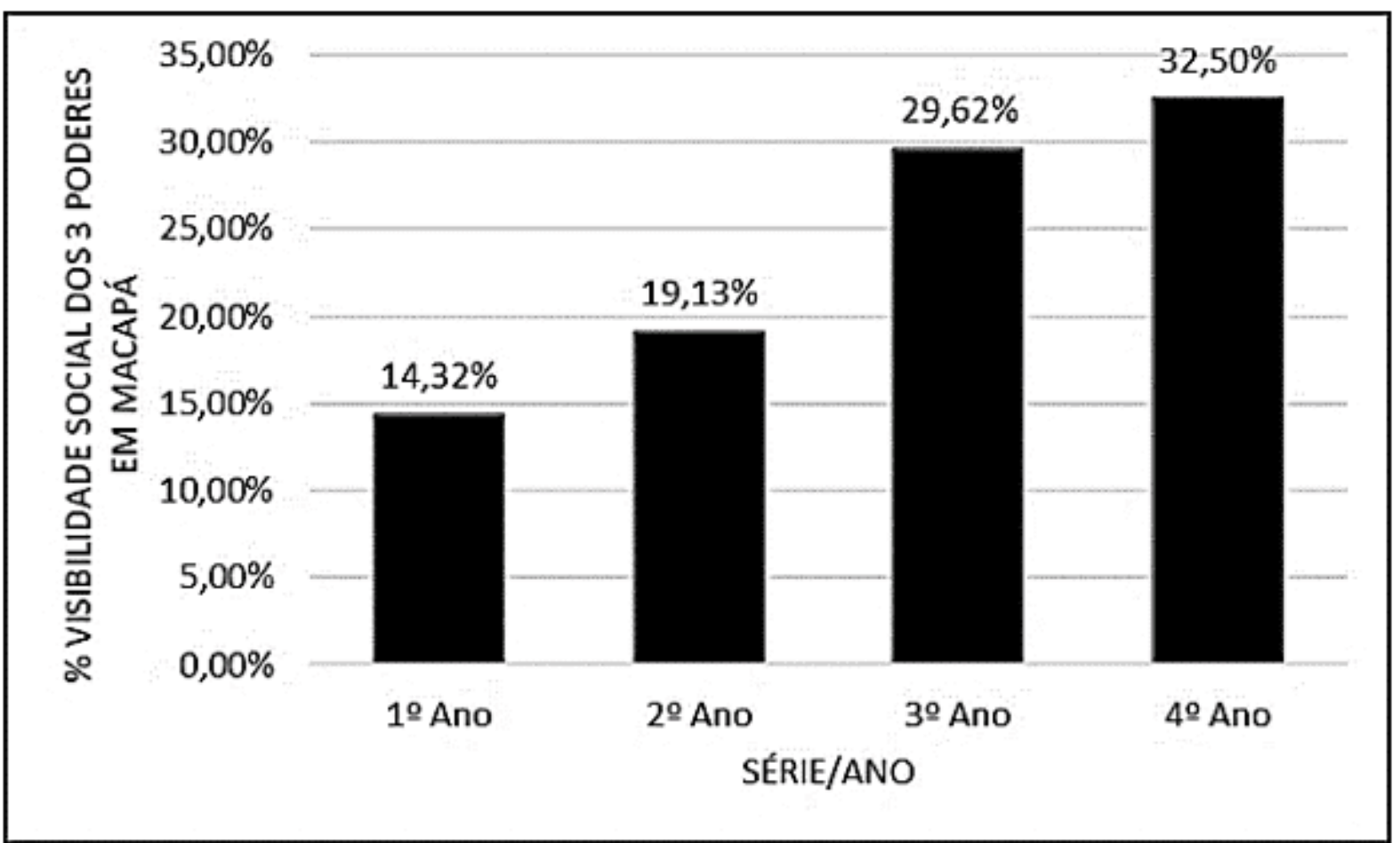

RC: 83562

Disponível em: https://www.nucleodoconhecimento.com.br/formazioneit/integralizzato-dellistituto 
Figura 16 Visibilità sociale dei tre poteri costituenti a Macapá in base all'età.

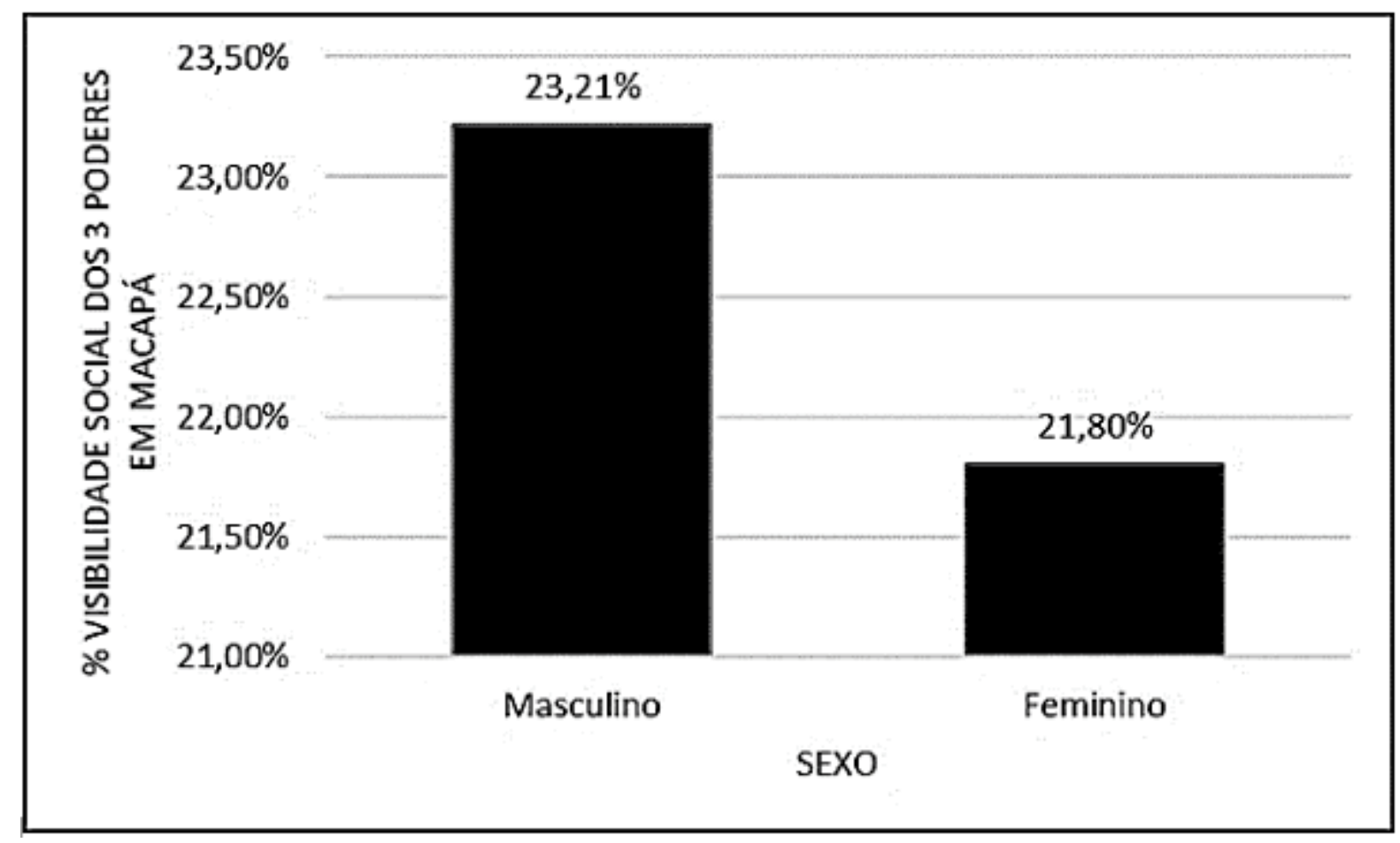

RC: 83562

Disponível em: https://www.nucleodoconhecimento.com.br/formazioneit/integralizzato-dellistituto 
Figura 17 Visibilità sociale dei tre poteri costituenti a Macapá in base all'età.

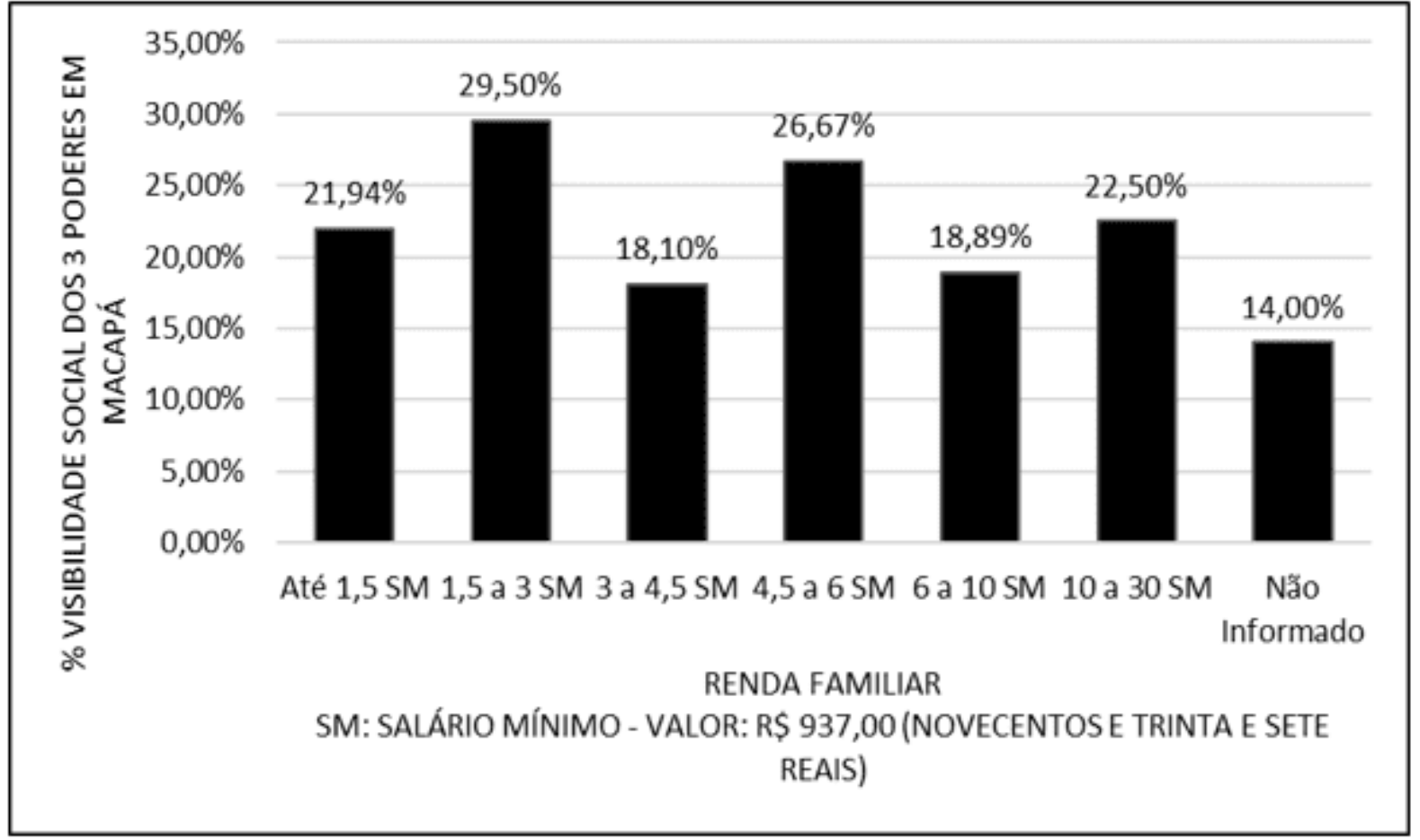

Questo risultato può essere dovuto alle conoscenze acquisite a scuola, con la famiglia o prima della società, che con la più grande interazione sociale che gli studenti acquisiscono nel corso della loro vita, finiscono per assimilare una maggiore quantità di informazioni sui poteri costituenti (SILVA FILHO, 2004).

La figura 18 mostra che il 49,01\% delle risposte scelte dagli intervistati si sbagliava, senza tenere conto della percentuale del 5,38\% delle risposte in bianco, che probabilmente i partecipanti non conoscevano la risposta corretta. Ciò dimostra che il testo costituzionale rimane una roccaforte degli esperti e che la stragrande maggioranza degli studenti probabilmente non ha mai nemmeno gestito la Costituzione federale, il che significa che non costituisce la realtà del liceo nella forma integralizzata. Non è lavorato, discusso dagli studenti. È quindi facile presumere che essi non siano certamente consapevoli dei loro diritti e doveri fondamentali espressi in quella paese. Se, quando completano il liceo, non padroneggiano tali conoscenze, resta da credere che verranno per tutta la vita dei

RC: 83562

Disponível em: https://www.nucleodoconhecimento.com.br/formazioneit/integralizzato-dellistituto 
loro cittadini, in occasioni di conflitto, o in altre più miti, se arrivano, quando un evento influisce sulla loro libertà o proprietà (DIAS e DE OLIVEIRA, 2015).

Figura 18: Esito delle domande sulle basi della Costituzione federale.

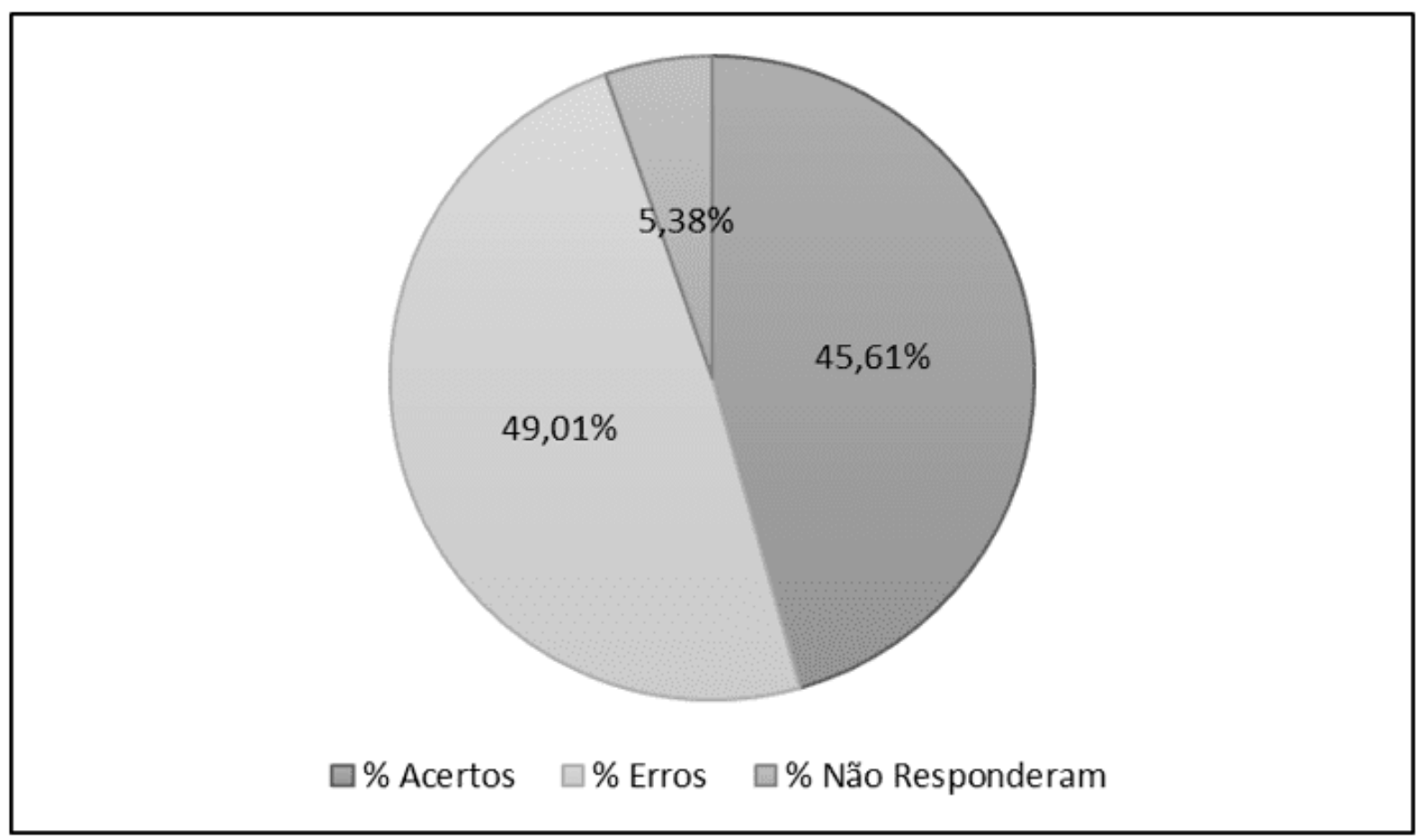

Le figure 19, 20, 21 e 22 che seguono mostrano i risultati delle domande nella sezione sulle basi della Costituzione federale in relazione rispettivamente ai fattori di età, grado/anno, sesso e reddito familiare. In questi risultati, abbiamo trovato la percentuale di risposte corrette in relazione alle risposte delle domande sul figlio di base di CF secondo i criteri ricercati. 
Figura 19 Percentuale di risposte corrette sulle basi della CF in base all'età.

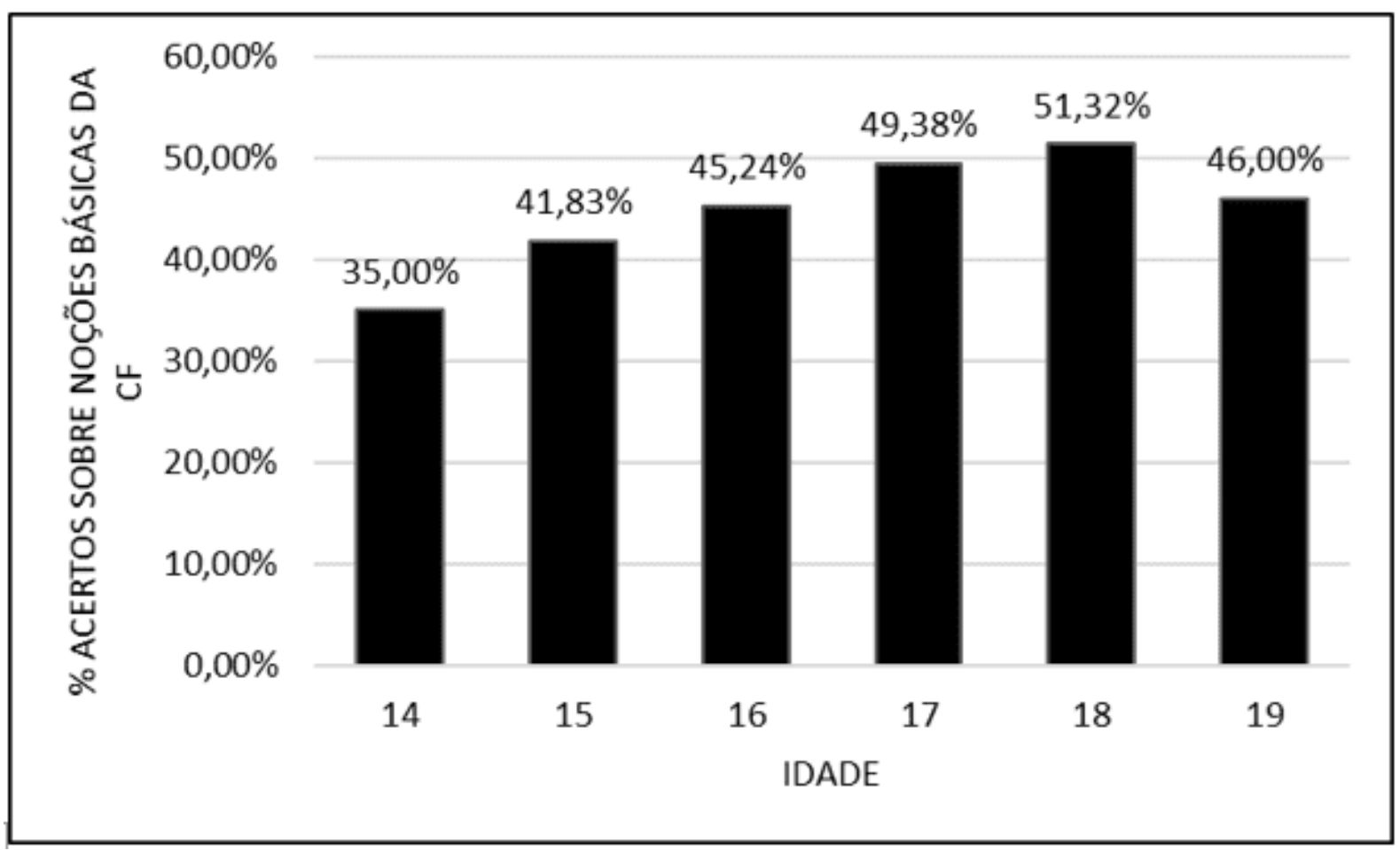

RC: 83562

Disponível em: https://www.nucleodoconhecimento.com.br/formazioneit/integralizzato-dellistituto 
Figura 20 Percentuale di risposte corrette sulle basi di CF in base alla serie/anno.

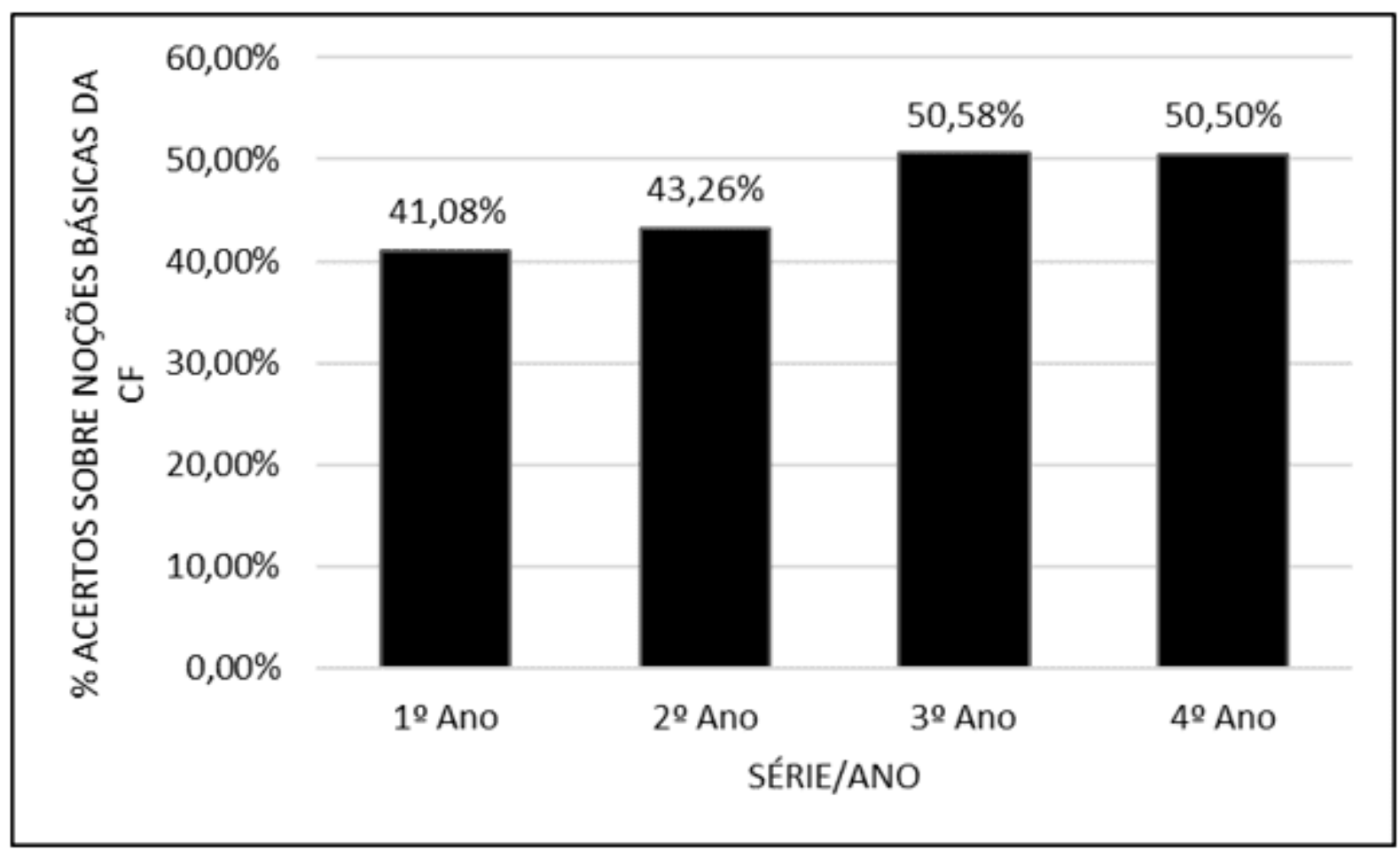

RC: 83562

Disponível em: https://www.nucleodoconhecimento.com.br/formazioneit/integralizzato-dellistituto 
Figura 21 Percentuale di risposte corrette sulle basi della CF in base al genere.

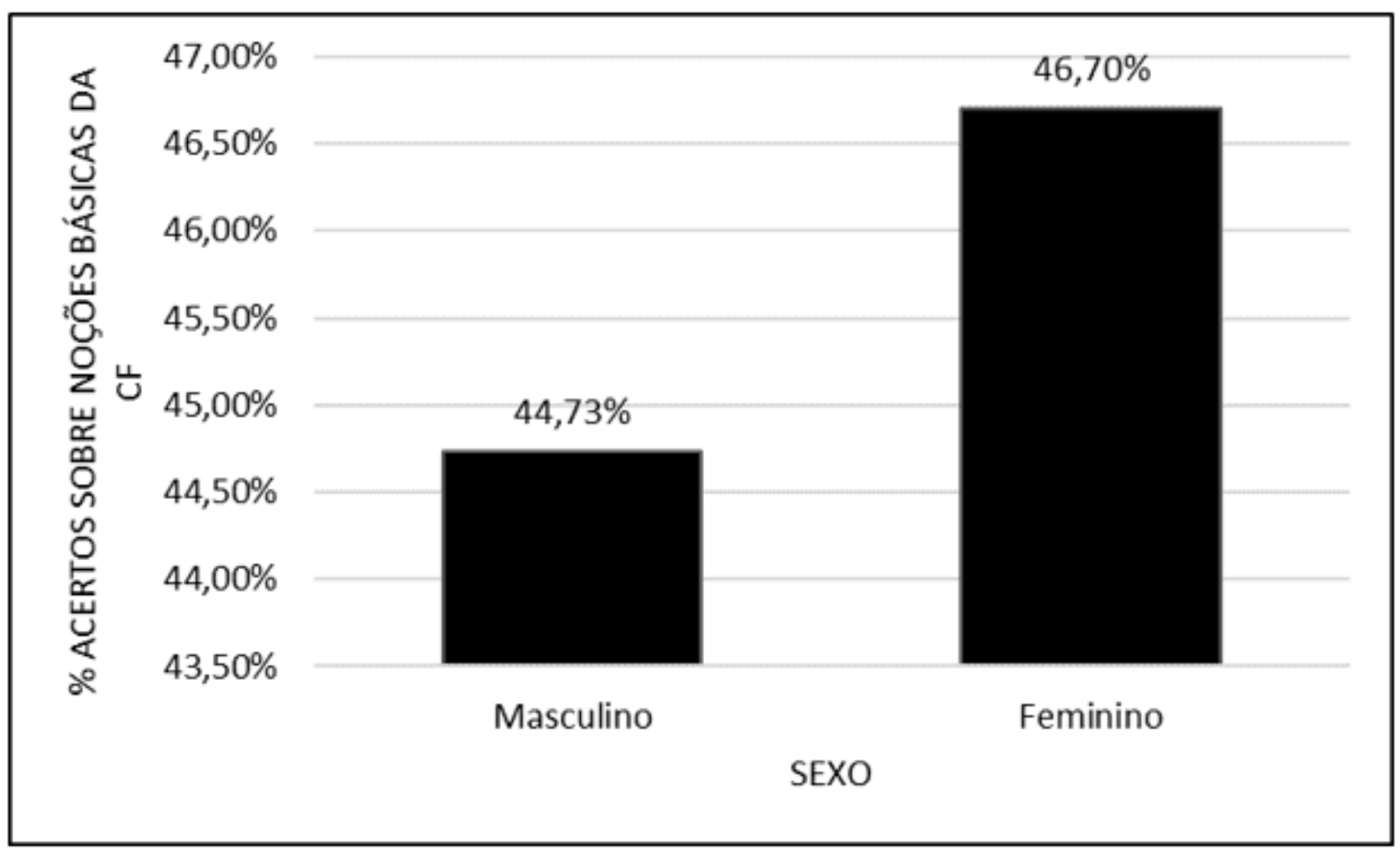

RC: 83562

Disponível em: https://www.nucleodoconhecimento.com.br/formazioneit/integralizzato-dellistituto 
Figura 22 Percentuale di risposte corrette sulle basi della CF in base al reddito familiare.

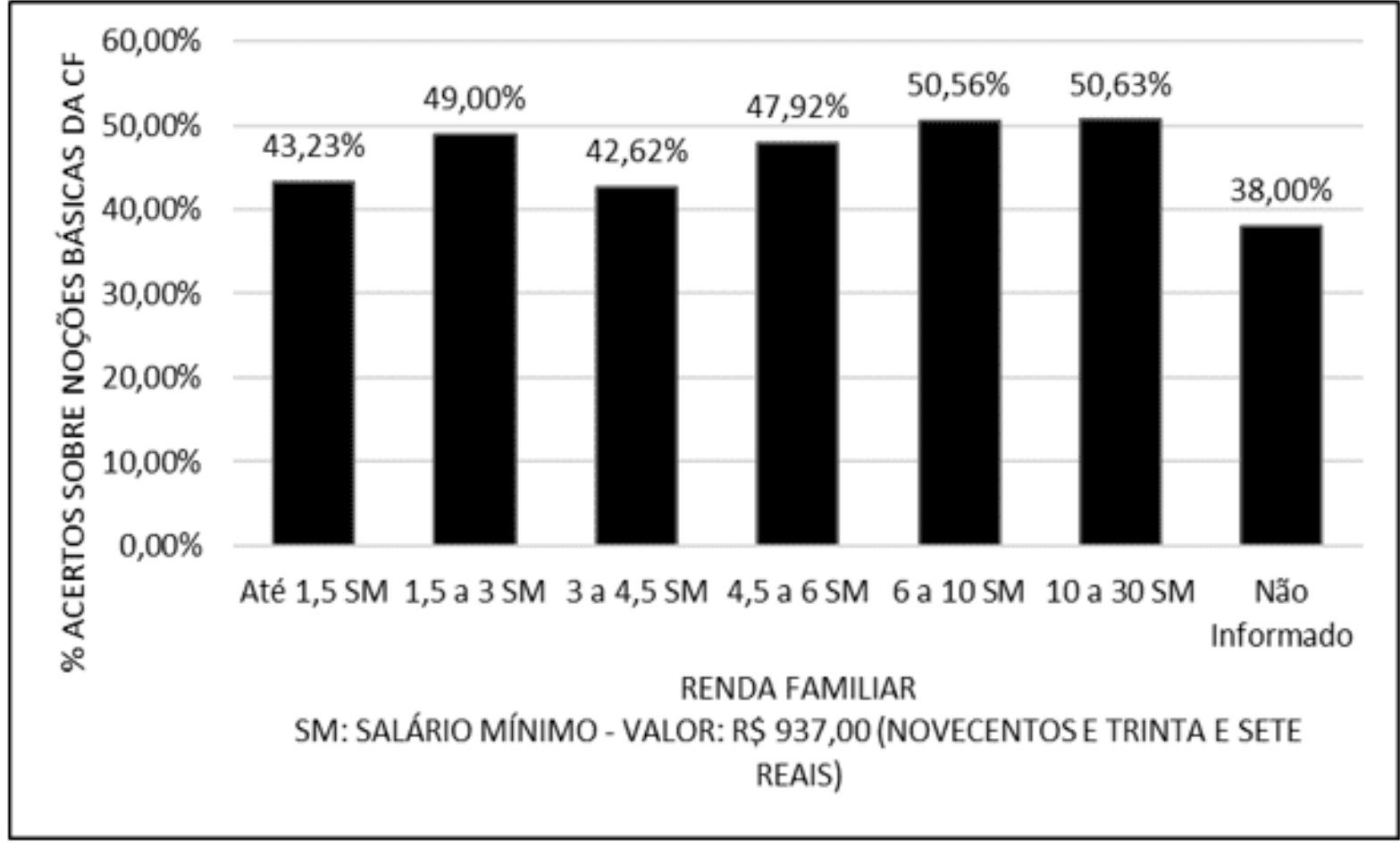

Sebbene i grafici delle figure 19 e 20 presentino alcune distorsioni nelle loro linee di tendenza, possiamo notare una percentuale più alta di risposte corrette man mano che gli studenti avanzano al voto successivo e, di conseguenza, hanno un'età più elevata, questo è il risultato di un'interazione sociale da parte degli intervistati. La distorsione delle ultime due colonne dei due grafici sopra menzionati riflette probabilmente l'insonous ity di questi studenti con il questionario o perché sono per lo più studenti ripetuti che non mostrano interesse per il processo di insegnamento e apprendimento dell'istituto (ALMEIDA e ALVES, 2021).

Possiamo anche notare nella figura 21 un equilibrio nelle risposte corrette degli intervistati maschi e femmine, il che riflette che questo fattore non interferisce direttamente nei risultati ottenuti. Per quanto riguarda la figura 22, la Corte ha riscontrato una leggera tendenza degli intervistati con un reddito familiare più elevato a presentare un tasso più elevato di risposte corrette, ma questa influenza non è

RC: 83562

Disponível em: https://www.nucleodoconhecimento.com.br/formazioneit/integralizzato-dellistituto 
molto significativa e può essere facilmente messa in discussione (FERREIRA e LEISMANN, 2020)

II risultato della figura 23 mostra che la maggior parte degli intervistati dimostra di avere principi di giustizia e filosofia essenziali per l'interazione sociale, non presentando contraddizioni nei loro principi, anche se rileviamo alcune risposte contraddittorie e vuote da parte di alcuni intervistati.

Figura 23 Percentuale di studenti che dimostrano di avere un certo principio di giustizia e filosofia. Figura 24 Percentuale di studenti che hanno risposto alle risposte con influenza della loro vita sociale.

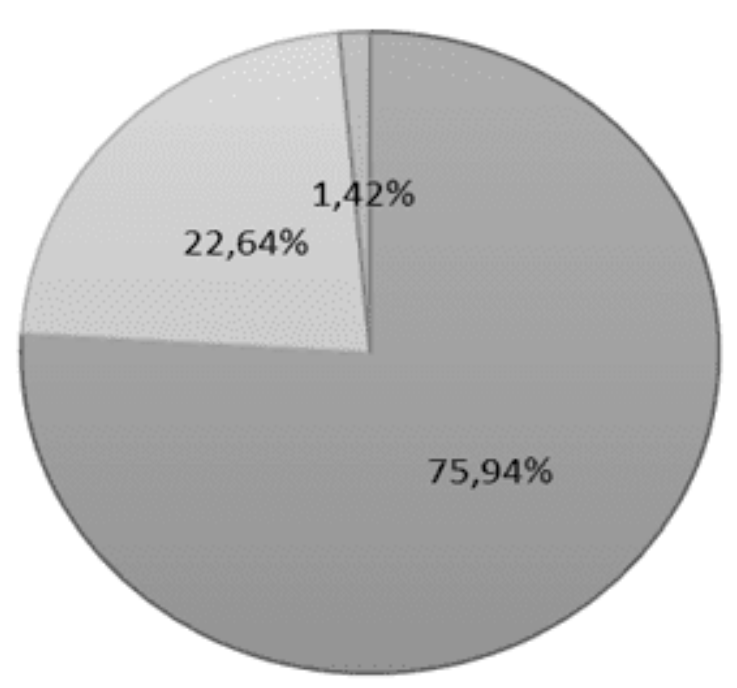

$\square$ Demonstram Possuir $\square$ Não Demonstram Possuir $\square \%$ Não Responderam

RC: 83562

Disponível em: https://www.nucleodoconhecimento.com.br/formazioneit/integralizzato-dellistituto 
La figura 24 mostra che pochi studenti intendono frequentare la legge, questo era già previsto, dal momento che gli intervistati frequentano gli edificazioni, corso nell'area esatta, riflettendo che l'intenzione di frequentare la legge non ha influenzato in modo significativo le risposte ottenute.

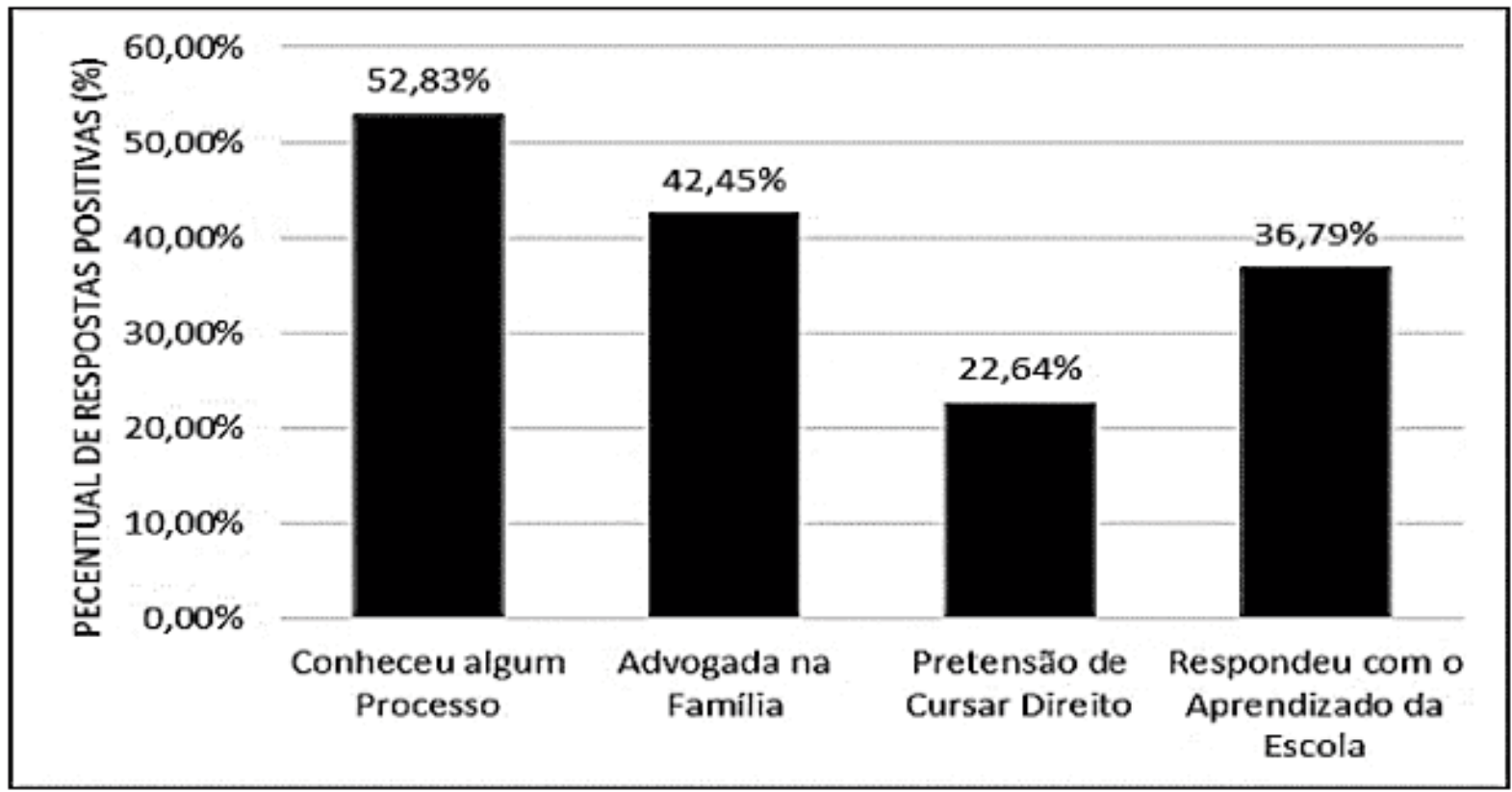

D'altro canto, mostriamo che il 52,83 per cento degli intervistati ha già assistito a un processo giudiziario in una fase della loro vita, che può aver contribuito alle loro risposte, questo è un avvertimento per la società, perché è sempre più essenziale che i cittadini abbiano una conoscenza di base del diritto, poiché avranno bisogno di questa conoscenza nella loro vita quotidiana (LOTTERMANN et al., 2020).

Il punto negativo è che pochi studenti hanno confermato di aver risposto alle domande del questionario con ciò che hanno imparato a scuola, dimostrando un fallimento nell'insegnamento, poiché la funzione sociale della scuola è la propagazione e la democratizzazione delle conoscenze necessarie per la formazione di cittadini consapevoli, partecipativi e attivi nella società, e avendo come principi e obiettivi della Repubblica Federativa del Brasile nella costruzione di una società libera, equo e solidale (LOTTERMANN et al., 2020). 
Sebbene la figura 25 mostri che solo il 35,85\% degli studenti intervistati ha fatto qualche commento, tutti i commenti scritti in questa sezione, in generale, sono stati positivi. Tra i commenti degli studenti c'era un'omogeneità di genere e per le classi più avanzate, abbiamo sempre ottenuto un numero minore di commenti.

Figura 25 Percentuale di studenti che hanno commentato il questionario.

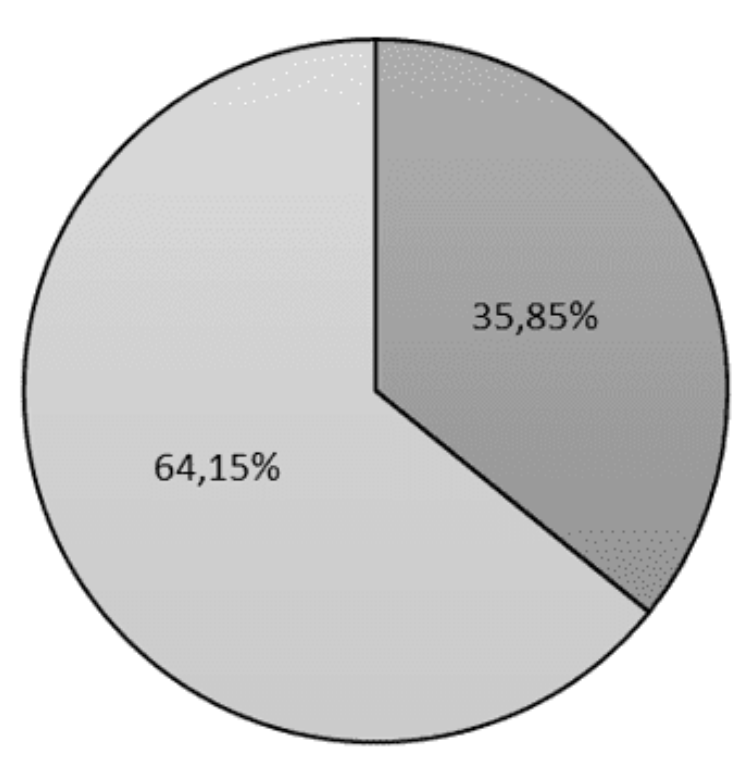

$\square \%$ Comentários $\quad \square \%$ Sem Comentários

La maggior parte dei commenti registrati ha riferito che il questionario era molto interessante e ben elaborato, a conferma della pertinenza dello studio CF nelle scuole. Abbiamo anche rilevato che alcuni studenti si sono svegliati con la mancanza di conoscenza della materia, segnalando di non aver mai imparato questo a scuola (MORAES, 2013).

Abbiamo avuto alcuni commenti inaspettati, come il fatto che uno studente non risiedeva nel comune di Macapá, ostacolando le sue risposte sulla posizione spaziale dei poteri. Un altro commento ha affermato che il questionario ha suscitato un maggiore apprezzamento dello studente per il corso di giurisprudenza, al quale 
intende partecipare, per non parlare dei giochi, tipici degli studenti di questa fascia d'età, usando parole come: "top", ten, ecc., per descrivere il questionario

\section{CONCLUSIONI}

Nonostante i diversi progetti finora realizzati in relazione all'attuazione della legge nelle scuole di base, questo sforzo non è ancora riuscito a raggiungere l'intera nazione brasiliana, ma è uno sforzo da praticare con responsabilità e speranza sulla strada verso una trasformazione sociale.

La buona volontà della maggior parte delle materie coinvolte nel cambiamento della realtà brasiliana è stata percepita insegnando una disciplina di vitale importanza. Ciò è stato verificato nei risultati dei questionari, ritenendo che la loro domanda fosse piuttosto salutare, perché ha suscitato dibattiti e dubbi tra gli interessati, che si sono conclusi, in un certo senso, qualificando i partecipanti.

Il liceo brasiliano, esaminato da un taglio puntuale del corso tecnico negli edifici nella forma integralizzata dell'IFAP, campus Macapá, non può rispettare le raccomandazioni del CF e della LDB in base al risultato del questionario applicato agli studenti. Questo insegnamento occupa un posto privilegiato nell'educazione educativa brasiliana, tra le scuole elementari e le.

Gli studenti dimostrano di essere entusiasti della possibilità di implementare scansioni di base nel corso tecnico negli edifici dell'IFAP, nel campus di Macapá, anche se facoltativamente.

\section{RIFERIMENTI}

ALMEIDA, F. A.; ALVES, M. T. G. A cultura da reprovação em escolas organizadas por ciclos. Revista Brasileira de Educação, v. 26, p. 1-28, 2021.

$\mathrm{RC}: 83562$

Disponível em: https://www.nucleodoconhecimento.com.br/formazioneit/integralizzato-dellistituto 
AMARAL, D. P. D.; CASTRO, M. M. D. Educação moral e cívica: A retomada da obrigatoriedade pela agenda conservadora. Cad. Pesqui., v. 50, n. 178, p. 10781096, 2020.

ANTONELLO, I. P.; NOREMBERG, A. DIREITO E EDUCAÇÃO: a promoção da cidadania nas escolas brasileiras utilizando-se da disciplina de direito constitucional. XII Seminário De Demandas Sociais E Políticas Públicas Na Sociedade Contemporânea. Santa Cruz do Sul RS: UNISC 2016.

BRASIL. LEI № 8.112, DE 11 DE DEZEMBRO DE 1990. Brasilia DF, 1990. Disponível em: < http://www.planalto.gov.br/ccivil_03/leis//8112cons.htm >. Acesso em: 17 mar 2021.

. Constituição da República Federativa do Brasil (1988). DEPUTADOS, C.

D. Brasília DF: Edições Câmara 2012.

. LDB: Lei de diretrizes e bases da educação nacional - Lei no 9.394, de 20 de dezembro de 1996. Brasília DF: Edições Câmara 2016.

. Novo Ensino Médio - DÚVIDAS. MEC. Brasilia DF: MEC 2017.

DIAS, L. S.; DE OLIVEIRA, L. B. Acesso à educação jurídica: pela inclusão do ensino jurídico na grade curricular do ensino regular. Rio de Janeiro RJ, 2015. Disponível em:

https://periodicos.ufrn.br/constituicaoegarantiadedireitos/article/viewFile/8159/5897 >. Acesso em: 13 jun. 2017.

FERREIRA, T. A.; LEISMANN, E. L. Lei anticorrupção: Conhecimento e percepção na academia a partir de diferentes condições socioeconômicas. Administração de Empresas em Revistas v. 2, n. 21, p. 216-234, 2020. 
FIGUEIREDO, C. B. D. As Funções Do Estado: O Princípio Da Separação De Poderes. XVIII Jornada e Mostra de Estudos Jurídicos e Sociais. Santa Rosa RS: Faculdades Integradas Machado De Assis: 44-48 p. 2019.

FONSECA, C. J. D.; FERNANDES, L. F. A importância do conhecimento contábil matemático para a ciência jurídica: Uma revisão integrativa. J Business Techn, v. 17, n. 3, p. 116-135, 2020.

HOHENDORFF, R. V. et al. "Você Sabia? O Direito te Desafia!": Relato de Caso do Uso de Jogo de Tabuleiro com Cartas para Demonstrar a Alunos de Ensino Médio o Quanto o Direito pode ser Instigante. Anais do V Congresso sobre Tecnologias na Educação (Ctrl+E 2020). João Pessoa PB 2020.

IBGE. População - Distribuição da população por sexo. Brasilia DF, 2010. Disponível em: < https://brasilemsintese.ibge.gov.br/populacao/distribuicao-dapopulacao-por-sexo.html >. Acesso em: 17 mar 2021.

. Contas nacionais - PIB per capita. Brasilia DF, 2021. Disponível em: < https://brasilemsintese.ibge.gov.br/contas-nacionais/pib-per-capita.html >. Acesso em: 17 mar 2021.

IFAP. Resolução no 67.2019 Consup. Macapa AP: IFAP 2019.

Histórico. Macapa AP, 2021. Disponível em: < https://www.ifap.edu.br/index.php/quem-somos/historico >. Acesso em: 17 mar 2021.

LORENSET, R. B. C. et al. Direito e linguagem se (entre)laçam e se (con)fundem. Anuário Pesquisa e Extensão Unoesc Xanxerê, v. 6, p. 1-6, 2021.

LOTTERMANN, O.; SIQUEIRA, E.; CESARO, I. A. D. Mediações Do Conhecimento Escolar De Ensino Médio Com O Conhecimento Científico, O Mundo Do Trabalho E O Exercício Da Cidadania. Contexto \& Educação, n. 112, p. 200-216, 2020.

RC: 83562

Disponível em: https://www.nucleodoconhecimento.com.br/formazioneit/integralizzato-dellistituto 
MORAES, E. D. F. R. M. A importância da introdução de disciplinas jurídicas no ensino médio. Magistro, v. 8, n. 2, p. 27-45, 2013. Disponível em: < http://publicacoes.unigranrio.edu.br/index.php/magistro/article/view/2119/997 >.

NÓBREGA, V. V. D.; CORRÊA, C. S.; JESUS, J. C. D. Diferenciais De Gênero No Mercado De Trabalho Brasileiro Em 2016 E 2017. Campinas, SP, 2019. Disponível em: < https://abep.org.br/publicacoes/index.php/anais/article/viewFile/3556/3407 >. Acesso em: 17 mar 2021.

ROMARIO. Projetos de Lei. Brasilia DF, 2021. Disponível em: < https://romario.org/projetos/lei/ >. Acesso em: 17 mar 2021.

SARAIVA. Vade Mecum. 11. São Paulo SP: Saraiva, 2017.

SASSE, C. Pesquisa DataSenado mostra que poucos conhecem realmente a Constituição. 2013.

Disponível em:

http://www12.senado.leg.br/noticias/materias/2013/10/25/pesquisa-datasenadomostra-que-poucos-conhecem-realmente-a-constituicao-do-pais >. Acesso em: 4 out. 2017.

SILVA FILHO, N. A. Projeto OAB Vai à Escola continua se expandindo. São Paulo SP, 2004. Disponível em: < http://www.oabsp.org.br/noticias/2004/05/18/2413 >. Acesso em: 22 nov. 2017.

TAVARES, T. P.; MOTA, M. F.; FELDENS, D. G. O Ensino Moral E Cívico Como Princípios Educativos Na Formação Dos Cidadãos. Interfaces Científicas v. 8, n. 3, p. 422-433, 2020.

Inviato: aprile 2021.

Approvato: aprile 2021. 